\title{
Religiöse Rechtfertigung? Die Beschneidung von Knaben zwischen Strafrecht, Religionsfreiheit und elterlichem Sorgerecht
}

\author{
Bijan Fateh-Moghadam*
}

A. Rechtskulturen: Die Beschneidung von Knaben im Horizont der Strafrechtswissenschaft...................... 115

B. Beschneidungskulturen: rechtssoziologische Grundlagen................. 117

I. Die Beschneidung von Knaben als mehrdeutige soziale Praxis .......... 118

II. Globale Prävalenz der Zirkumzision .............................. 120

C. Die strafrechtliche Legitimation der Beschneidung von Knaben ........... 121

I. Körperverletzungsdoktrin .......... 121

II. Die Einwilligung des Minderjähri-

gen ............................ 122

1. Objektive Schranken der Einwilligung in die Zirkumzision ....... 123

2. Einwilligungsfähigkeit und medizinische Indikation ............ 124

3. Vetorechte nicht einwilligungsfähiger Minderjähriger ........... 126

III. Stellvertretende Einwilligung in die Zirkumzision ...................... 127

1. Die stellvertretende Einwilligung als Einwilligung des Verletzten. . 128
2. Best interest versus substituted judgement - rechtsvergleichende Überlegungen ................... 129

3. Verfassungsrechtliche Garantie der stellvertretenden Einwilligung ......................... 131

4. Der Missbrauch der Dispositionsbefugnis als strafrechtliche Grenze der stellvertretenden Einwilligung .................... 133
a) Die Schwere des Eingriffs, seine gesundheitlichen Folgen und Risiken................ 134
b) Kurativ- und präventivmedi- zinische Vorteile sowie sons- tige Vorteile und Chancen... 135
c) Das Fehlen von spezifisch kindeswohlverletzenden Ein- griffsmodalitäten........... 138
d) Ergebnis .................. 138

D. Religionsfreiheit und Zirkumzision . . 139

I. Religiöse Rechtfertigung?........... 139

II. Religionsfreiheit als Abwehrrecht . . 140

E. Fazit ............................. 141

\section{A. Rechtskulturen: Die Beschneidung von Knaben im Horizont der Strafrechtswissenschaft}

In der deutschen Strafrechtswissenschaft wird die Frage der Strafbarkeit der Beschneidung (med.: Zirkumzision) von männlichen Minderjährigen kontrovers diskutiert. ${ }^{1}$ Die noch junge Debatte beendet eine Phase der juristischen Selbstbeschränkung, in der die Praxis der Beschneidung von Knaben bemerkenswerterweise überhaupt nicht als Rechtsproblem thematisiert wurde. Ein Grund für das neue Interesse des Strafrechts an der Zirkumzision dürfte dabei darin bestehen, dass die Thematik

* Akad. Rat Dr. Bijan Fateh-Moghadam ist Projektwissenschaftler im Exzellenzcluster Religion und Politik, Teilprojekt „Normenbegründung im pluralistischen Staat“, der Westfälischen Wilhelms-Universität Münster mit Auftrag zur Lehre für Medizinstrafrecht und Rechtssoziologie. Der Verfasser dankt den Veranstaltern und Teilnehmern des Kriminalwissenschaftlichen Kolloquiums der Universität Münster sowie des Forums Junge Rechtswissenschaft der Universität Tübingen für die Diskussion einer Vortragsfassung des vorliegenden Beitrags.

1 Zum Diskussionsstand vgl. die Nachweise bei T. Fischer, Strafgesetzbuch und Nebengesetze, 57. Aufl., München 2010, $\$ 223$ Rn. 6 b sowie unten Fn. 3-6; aus verfassungsrechtlicher Sicht W. Höfling, $\mathbb{1 5 5}$ Elternrecht, in: J. Isensee/P. Kirchhof (Hrsg.), Handbuch des Staatsrechts der Bundesrepublik Deutschland, Band 7 Freiheitsrechte, Heidelberg 2009, S. 477 Rn. 85; aus familienrechtlicher Sicht M. Coester, in: M. Coester/H. Engler/L. Salgo (Hrsg.), J. von Staudingers Kommentar zum Bürgerlichen Gesetzbuch mit Einführungsgesetz und Nebengesetzen, Buch 4, Familienrecht, $\mathbb{S} 1638-1683$, Berlin 2004, $\$ 1666$ Rn. 163. 
als ein weiterer Ausdruck der Folgeprobleme religiöser Pluralisierung wahrgenommen wird, die gegenwärtig verstärkt die Rechtswissenschaft beschäftigen. ${ }^{2}$ So fokussieren sowohl Befürworter als auch Gegner der Kriminalisierung der Zirkumzision vor allem die strafrechtlichen Grenzen der Religionsfreiheit. In der Strafrechtswissenschaft mehren sich die Stimmen, die jede medizinisch nicht zwingend notwendige Beschneidung von minderjährigen Knaben ungeachtet der Einwilligung der Personensorgeberechtigten als rechtswidrige Körperverletzung qualifizieren. ${ }^{3}$ Strenge Anforderungen an das Vorliegen einer medizinischen Indikation, die selbst in Fällen diagnostizierter Vorhautverengung (Phimose) nicht mehr vorliegen soll, wenn eine alternative, konservative Behandlungsmethode in Betracht kommt, ${ }^{4}$ sowie die Regelannahme, dass Minderjährige nicht die erforderliche Einsichts- und Urteilsfähigkeit zur Einwilligung in die Beschneidung besitzen, ${ }^{5}$ führen nach diesem Ansatz zu einer nahezu vollständigen Kriminalisierung der Zirkumzision von Knaben unter 18 Jahren. Die Vertreter der Strafbarkeitsthese wenden sich dabei insbesondere gegen die Konstruktion einer religiösen Rechtfertigung nicht kurativ-medizinisch indizierter Zirkumzisionen. Genau dies, eine religiöse Rechtfertigung im Sinne eines partikularen Erlaubnissatzes für religiös motivierte Beschneidungen, versucht die Gegenansicht zu begründen, indem sie die Bedeutung der Religionsfreiheit in den Mittel-

2 Vgl. nur H. Dreier, Religion und Verfassungsstaat im Kampf der Kulturen, in: H. Dreier/E. Hilgendorf (Hrsg.), Kulturelle Identität als Grund und Grenze des Rechts, Akten der IVR-Tagung vom 28.-30. September 2006 in Würzburg, Stuttgart 2008, S. 11; H. M. Heinig/M. Morlok, Von Schafen und Kopftüchern, Das Grundrecht auf Religionsfreiheit in Deutschland vor den Herausforderungen religiöser Pluralisierung, JZ 2003, S. 777; E. Hilgendorf, Strafrecht und Interkulturalität, Plädoyer für eine kulturelle Sensibilisierung der deutschen Strafrechtsdogmatik, JZ 2009, S. 139; T. Hörnle, Strafrechtliche Verbotsnormen zum Schutz von kulturellen Identitäten, in: Dreier/Hilgendorf (Hrsg.), Identität (Fn. 2), S. 315; M. Rohe, Islamisierung des deutschen Rechts?, JZ 2007, S. 801; U. Sacksofsky, Religiöse Freiheit als Gefahr?, 1. Bericht, in: C. Engel (Hrsg.), Erosion von Verfassungsvoraussetzungen, Berichte und Diskussionen auf der Tagung der Vereinigung der Deutschen Staatsrechtslehrer in Erlangen vom 1. bis 4. Oktober 2008, Berlin 2009, S. 7; C. Walter, Religiöse Freiheit als Gefahr? Eine Gegenrede, DVB1 2008, S. 1073

3 R. D. Herzberg, Rechtliche Probleme der rituellen Beschneidung, JZ 2009, S. 332; G. Jerouschek, Beschneidung und das deutsche Recht - Historische, medizinische, psychologische und juristische Aspekte, NStZ 2008, S. 313; H. Putzke, Die strafrechtliche Relevanz der Beschneidung von Knaben, Zugleich ein Beitrag über die Grenzen der Einwilligung in Fällen der Personenfürsorge, in: H. Putzke/B. Hardtung/ T. Hörnle/R. Merkel et al. (Hrsg.), Strafrecht zwischen System und Telos, Festschrift für Rolf Dietrich Herzberg zum 70. Geburtstag am 14. Februar 2008, Tübingen 2008, S. 669; H. Putzke, Rechtliche Grenzen der Zirkumzision bei Minderjährigen, MedR 2008, S. 268; i.E. wohl auch D. Sternberg-Lieben, Die Strafbarkeit eines nicht indizierten ärztlichen Eingriffs, in: M. Böse/D. Sternberg-Lieben (Hrsg.), Grundlagen des Straf- und Strafverfahrensrechts, Festschrift für Knut Amelung zum 70. Geburtstag, Berlin 2009, S. 325 (352 f. mit Fn. 186); a.A. wohl noch Fischer, Strafgesetzbuch (Fn. 1), $\mathbb{2 2 3}$ Rn. 6 b und Rohe, Islamisierung (Fn. 2), S. 805; offen gelassen bei H.-U. Paeffgen, in: U. Kindhäuser/U. Neumann/H.-U. Paeffgen (Hrsg.), Strafgesetzbuch, Bd. II, 3. Aufl., Baden-Baden 2010, $\$ 228$ Rn. 18 mit Fn. 49.

4 M. Stehr/H. Putzke/H.-G. Dietz, Zirkumzision bei nicht einwilligungsfähigen Jungen, Strafrechtliche Konsequenzen auch bei religiöser Begründung, Deutsches Ärzteblatt 2008, S. 1778 (1780).

5 Jerouschek, Beschneidung (Fn. 3), S. 318; Putzke, Beschneidung, in: Putzke et al. (Hrsg.), Strafrecht (Fn. 3), S. 669, S. 685 f. 
punkt ihrer Argumentation stellt. ${ }^{6}$ Die Betonung des religiösen Aspektes der Beschneidungspraxis führt zu einer Kulturalisierung der Problemstellung, die sich vermeiden lässt, wenn man die systematisch vorrangig zu klärenden Grenzen des elterlichen Sorgerechts genauer in den Blick nimmt. Eine verfassungsrechtlich informierte Rekonstruktion der Reichweite und Grenzen der stellvertretenden Einwilligung zeigt, dass die Strafrechtsdogmatik die Beschneidungsproblematik differenzierend lösen kann, ohne auf die zweifelhafte Konstruktion einer religiösen oder kulturellen Rechtfertigung ${ }^{7}$ zurück greifen zu müssen.

Ungeachtet der neueren rechtswissenschaftlichen Debatte über die Strafbarkeit der Beschneidung ist bisher weder in Deutschland noch in einem anderen europäischen Land eine Gerichtsentscheidung ergangen, die eine lege artis durchgeführte Beschneidung von Knaben mit Zustimmung der Sorgeberechtigten für strafbar erachtet hätte. ${ }^{8}$ Die Grenzen der Zulässigkeit der männlichen Beschneidung befinden sich indes auch in anderen europäischen Ländern, insbesondere in Dänemark, Finnland und Schweden in der rechtspolitischen Diskussion. ${ }^{9}$

\section{B. Beschneidungskulturen: rechtssoziologische Grundlagen}

Untersucht man die Praxis der Beschneidung von Knaben als Phänomen der gesellschaftlichen Wirklichkeit, so stößt man auf plurale Beschneidungskulturen, die die Entfernung der männlichen Vorhaut unterschiedlich deuten.

6 K.-A. Schwarz, Verfassungsrechtliche Aspekte der religiösen Beschneidung, JZ 2008, S. 1125; K. Zähle, Religionsfreiheit und fremdschädigende Praktiken, Zu den Grenzen des forum externum, AöR 2009, S. 434; in diese Richtung weisend auch W. Gropp, Strafrecht, Allgemeiner Teil, 3. Aufl., Berlin 2005, S. 231 .

7 Vgl. dazu A. D. Renteln, The Cultural Defense, Oxford 2004; T. Frischknecht, "Kultureller Rabatt", Überlegungen zu Strafausschluss und Strafermäßigung bei kultureller Differenz, Bern 2009; Hörnle, Verbotsnormen, in: Dreier/Hilgendorf (Hrsg.), Identität (Fn. 2), S. 315; aus verfassungsrechtlicher Perspektive G. Britz, Kulturelle Rechte und Verfassung, Über den rechtlichen Umgang mit kultureller Differenz, Tübingen 2000.

8 Zur Unwirksamkeit einer durch Täuschung erlangten Einwilligung der Eltern in eine rituelle Beschneidung, die von einem Nichtmediziner unter unsterilen Bedingungen und auch sonst behandlungsfehlerhaft durchgeführt wurde, vgl. LG Frankenthal MedR 2005, S. 243. Zur Verletzung des allgemeinen Persönlichkeitsrechts durch eine von einem nicht sorgeberechtigten Vater ohne Zustimmung der sorgeberechtigten Mutter veranlassten Beschneidung eines nicht einwilligungsfähigen Knaben vgl. OLG Frankfurt NJW 2007, S. 3580.

9 J. Schiratzki, Banning God's law in the name of the Holy Body - The Nordic position on ritual male circumcision, Draft of speech at ISFL Regional Conference "Family Law in a Multicultural Environment: Civil and Religious Law in Family Matters", June 7-9, 2009, http://www.law2.byu.edu/isfl/israelcon2009/drafts/Johanna\%20Schiratzki.pdf. 


\section{Die Beschneidung von Knaben als mehrdeutige soziale Praxis}

Die kulturelle Dimension der männlichen Beschneidung erschöpft sich nicht in ihrer die Diskussion dominierenden religiösen Bedeutung im Islam und im Judentum. ${ }^{10}$ Die Vielschichtigkeit der sozialen Praxis der Zirkumzision lässt sich dabei nicht zuletzt an Phänomenen der Popkultur und ihrer medialen Inszenierung ablesen. So wird der Umstand, dass modern-säkulare weibliche Singles in New York ausnahmsweise auch auf unbeschnittene Männer treffen können, in der international erfolgreichen US-amerikanischen Fernsehserie "Sex and the City“ als ein ästhetisches Problem verhandelt, wobei unbeschnittene Männer tendenziell schlechter abschneiden als beschnittene. ${ }^{11}$ Die hier sichtbar werdende ästhetisch-kulturelle Dimension der Beschneidung kann ihrerseits vor dem Hintergrund der Medikalisierung der männlichen Beschneidung in den USA gelesen werden. Der US-amerikanische medizinische Diskurs säkularisiert die Praxis der Beschneidung, in dem er die (präventiv-)medizinischen Vorteile der Beschneidung betont und sie dadurch aus ihrem religiösen Kontext löst. ${ }^{12}$ Vor diesem Hintergrund kann sich eine ästhetische Beschneidungs(pop-)kultur ausbilden, die sich nach Maßgabe eigener Logiken weiter entwickelt. Der Dokumentarfilm „Partly Private. The Long Journey To A Short Cut" ${ }^{\text {13 }}$ hinterfragt den US-amerikanischen Pro-Beschneidungsmainstream, indem er den Entscheidungsfindungsprozess eines säkular-jüdischen Elternpaares nachzeichnet, das sich kontrovers mit der Frage der Beschneidung ihres Sohnes auseinandersetzt. Die Beschneidung wird hier als individual-ethisches Problem der Konsensfindung in Paar-Beziehungen diskutiert. Eine bemerkenswerte Perspektivenumkehr im Verhältnis von Tradition, Moderne und männlicher Beschneidung ergibt sich schließlich aus der jüngsten Entwicklung des internationalen präventiv-medizinischen Beschneidungsdiskurses. ${ }^{14}$ Im Rahmen der gegenwärtig durchgeführten Be-

10 Dazu Joint United Nations Programme on HIV/AIDS (UNAIDS), Male circumcision, Global trends and determinants of prevalence, safety and acceptability, 2007, S. 3 ff.; aus medizinhistorischer und beschneidungskritischer Sicht D. Gollaher, Das verletzte Geschlecht, Die Geschichte der Beschneidung, Berlin 2002, S. 13 ff. (zur jüdischen Tradition), S. 66 (zum Islam) und S. $51 \mathrm{ff.} \mathrm{(zum} \mathrm{frühen}$ Christentum).

11 Jenny Bicks (Author): Sex and the City, Season 2, Episode 9: „Old dogs, new dicks“. Es überrascht nicht, dass die TV-Serie von Anti-Beschneidungsaktivisten als mediale Verharmlosung kritisiert wird (H. Young, "That Thing": Portrayal of the Foreskin and Circumcision in Popular Media, in: G. Denniston/F. Hodges/M. F. Milos (Hrsg.), Circumcision and Human Rights, Dordrecht 2009, S. 239 (244)).

12 Zum Vergleich mit der Entwicklung im United Kingdom L. M. Carpenter, Theorizing Remedicalization: Routine Infant Male Circumcision in the United States and Great Britain, Paper presented at the American Sociological Association Annual Meeting, Sheraton Boston and the Boston Marriott Copley Place, Boston, MA, http://www.allacademic.com/meta/p241818_index.html, Stand vom: 23.1.2010.

13 Elon, Danae (Author/Director), Partly Private. The Long Journey To A Short Cut, Dokumentarfilm, Kanada 2009, ausgezeichnet auf dem Tribeca-Filmfestival, New York 2009.

14 Der medizinhistorischen Studie von Gollaher kommt das Verdienst zu, die historische Kontingenz medizinisch-wissenschaftlicher Paradigmen in Bezug auf die Beschneidung sichtbar zu machen (Gollaher, Geschlecht (Fn. 10)). Der Befund legt nahe, rechtliche Zuschreibungskriterien nicht unmittelbar an scheinbar deskriptiv-medizinische Kriterien wie die sogenannte medizinische Indikation zu koppeln. 
schneidungsprogramme zur HIV-Prävention der Weltgesundheitsorganisation (WHO) und der Vereinten Nationen (UNAIDS) im südlichen Afrika, ${ }^{15}$ wird die „kulturell bedingte Ablehnung der Zirkumzision"16 in einigen Bevölkerungsteilen zum präventiv-medizinischen Problem. Kulturelle Widerstände gegen die Beschneidung von Knaben und jungen Männern stellen eine Herausforderung für die kommunikativen Kompetenzen der professionellen Gesundheitsarbeiter dar, auf die ein Praxis-Leitfaden von UNAIDS vorbereiten soll:

„Complexity-sometimes including a long-standing opposition to the practice of male circumcision on cultural grounds - should not be a barrier to the initiation of dialogue. "17

Die in ihren kulturellen Traditionen verhafteten Beschneidungsgegner - so der Tenor des Leitfadens - müssen über die präventiv-medizinischen Vorteile der Beschneidung erst aufgeklärt werden, damit die moderne HIV-Präventionsstrategie erfolgreich umgesetzt werden kann.

Die Bedeutung von Beschneidungspraktiken, dies wird bereits an diesen wenigen Beispielen sichtbar, erschließt sich aus der Perspektive je spezifischer Sinnhorizonte, symbolischer Wissensordnungen und Codes. Diese Sinnhorizonte sind dabei nicht identisch mit dem, was der juristische Mainstream in der Regel meint, wenn von „Kultur und Recht“ die Rede ist, nämlich Kulturen im Sinne von homogenen kollektiven Gemeinschaften, etwa Ethnien, Völker, Nationen und Religionsgemeinschaften. ${ }^{18}$ Gemeint sind vielmehr religiöse, traditionelle, ästhetische, (präventiv-)medizinische, hygienische und schließlich auch juristische Sinnhorizonte, die ihren Gegenstand jeweils unterschiedlich konstruieren. Dies entspricht einem bedeutungsorientierten Kulturbegriff, für den die kultursoziologische Einsicht entscheidend ist, dass sämtliche Verhaltenskomplexe der Vergangenheit und Gegenwart, vom archaischen Ritus bis zur modernen Medizin, symbolische (Wissens-)Ordnungen voraussetzen, die den notwendigen handlungskonstitutiven Hintergrund aller sozialen Praktiken darstellen. ${ }^{19}$

Die individuellen Bestimmungsgründe (Determinanten) für die Entscheidung, seinen Sohn beschneiden zu lassen, wie sie sich aus subjektiver Sicht des Handelnden dar-

$15 \mathrm{Zu}$ den epidemiologischen Hintergründen vgl. unten C.III.4.b.

16 Joint United Nations Programme on HIV/AIDS (UNAIDS), Safe, voluntary, informed male circumcision and comprehensive HIV prevention programming, Guidance for decision-makers on human rights, ethical and legal considerations, 2008, S. 6.

17 Joint United Nations Programme on HIVIAIDS (UNAIDS), Prevention (Fn. 16), S. 6.

18 Sogenannter totalitätsorientierter Kulturbegriff (A. Reckwitz, Unscharfe Grenzen, Perspektiven der Kultursoziologie, Bielefeld 2008, S. 22 f.).

19 Reckwitz, Grenzen (Fn. 18), S. 25 ff. Zu den Grundlagen des bedeutungsorientierten Kulturbegriffs in Ernst Cassirers Philosophie der symbolischen Formen vgl. Reckwitz, Grenzen (Fn. 18), S. 26 sowie S. Moebius, Kultur, Bielefeld 2009, S. 19. 
stellen, können unterschiedliche Elemente dieser Sinnhorizonte aufnehmen und zu einem Motivbündel vereinen. Die aus beruflichen Gründen in Deutschland lebende, nicht religiöse amerikanische Familie, das ebenfalls religiös unmusikalische deutschiranische Ehepaar sowie deutsche Eltern jüdischen Glaubens können sich aus unterschiedlichen Gründen für oder gegen die Beschneidung ihrer Söhne entscheiden. Dabei müssen religiöse Motive keine Rolle spielen, und auch dort wo dies der Fall ist, können weitere Aspekte, etwa der jeweilige Stand der präventiv-medizinischen Wissenschaft, ästhetische und traditionelle Überlegungen für die Entscheidung von Bedeutung sein. Die hier vorgeschlagene Umstellung von einem totalitätsorientierten zu einem bedeutungsorientierten Kulturbegriff entzieht die juristische Diskussion der Beschneidung von Knaben der Kulturkampf-Rhetorik, die dieses Thema häufig belastet.

\section{Globale Prävalenz der Zirkumzision}

Die Mehrdeutigkeit der Praxis der Beschneidung lässt sich auch an den zur Verfügung stehenden empirischen Daten über die weltweite Prävalenz der Beschneidung ablesen. Die Zirkumzision gilt als die häufigste Operation im Kindesalter. ${ }^{20} \mathrm{Nach}$ den Angaben der WHO sind weltweit ca. $33 \%$ aller Männer über 15 Jahre beschnitten. ${ }^{21}$ Die Statistik erzeugt dabei ihrerseits eine spezifische Bedeutung der Beschneidung, indem sie sich an dem verfügbaren Datenbestand orientiert, der typischerweise nach Nationen und Religionsgruppen differenziert. Da Zirkumzisionen vielfach nicht systematisch statistisch erfasst werden, bedient sich die WHO indirekter Methoden der Datenkonstruktion. Sie unterstellt zunächst, dass alle muslimischen und jüdischen Männer beschnitten werden und addiert die verfügbaren Daten über „nicht-religiöse Beschneidungen“, die jedoch nur für einige Länder mit relativ hoher Prävalenz nicht-religiöser Beschneidungen statistisch gut dokumentiert sind. ${ }^{22}$ Als nicht-religiös gilt eine Beschneidung dabei - eine weitere bemerkenswerte statistische Vereinfachung - sofern sie nicht bei Moslems oder Juden durchgeführt wird. Die Gruppe der „nicht-religiösen Beschneidungen“ umfasst somit so heterogene Praktiken wie traditionelle bzw. rituelle Beschneidungen im südlichen Afrika, religiös motivierte Beschneidungen der koptischen Christen in Ägypten und der orthodoxen Christen in Äthiopien sowie die vor allem präventiv-medizinisch, ästhetisch und sozial motivierte Beschneidungspraxis in den USA, Kanada und dem Vereinigten Königreich. Besonders bemerkenswert ist die hohe Prävalenz nicht-religiöser

20 P. Malone/H. Steinbrecher, Medical aspects of male circumcision, British Medical Journal 2007, S. 1206 (1206).

21 Joint United Nations Programme on HIV/AIDS (UNAIDS), Circumcision (Fn. 10), S. 7.

22 Die Liste der Länder mit statistisch nachgewiesener hoher Prävalenz nicht-religiöser Beschneidungen umfasst Angola, Äthiopien, Australien, Ghana, Indonesien, Kanada, Kenia, Demokratische Republik Kongo, Republik Korea (Südkorea), Madagaskar, Nigeria, Philippinen, Südafrika, Uganda, United Kingdom, USA und Tanzania; Joint United Nations Programme on HIV/AIDS (UNAIDS), Circumcision (Fn. 10), S. 7 und 8 mit Tafel 1. 
Beschneidung in den USA, wo nach Angaben der WHO 75 \% aller Männer beschnitten sind. Als einziges europäisches Land ist das Vereinigte Königreich mit $6 \%$ erfasst. Addiert man die Zahlen von religiösen, also muslimischen und jüdischen und den gemäß der Länderliste der WHO erfassten nicht-religiösen Beschneidungen, ergibt sich ein (geschätzter) Anteil von $30 \% .{ }^{23}$ Unterstellt man, dass auch in Ländern, die von der WHO nicht selbständig erfasst werden konnten, also etwa auch in Deutschland, zumindest $5 \%$ der Männer aus nicht-religiösen Gründen beschnitten werden, erhöht sich der Anteil auf mindestens $33 \%{ }^{24}$

\section{Die strafrechtliche Legitimation der Beschneidung von Knaben}

Auf der Basis der Körperverletzungsdoktrin des Medizinstrafrechts (I.) kommen für die Legitimation der Beschneidung die Einwilligung des Minderjährigen selbst (II.) und die stellvertretende Einwilligung der Personensorgeberechtigten (III.) in Betracht. Die Frage nach den Grenzen der Dispositionsbefugnis der Sorgeberechtigten bildet dabei den Schwerpunkt des Beitrags. Zu den Grenzen der Dispositionsbefugnis gehört auch die Beachtung etwaiger Vetorechte nicht einwilligungsfähiger Minderjähriger. Schließlich ist die Frage nach der Bedeutung der Religionsfreiheit zu beantworten (IV.).

\section{Körperverletzungsdoktrin}

Bei der Zirkumzision von Knaben wird die Penisvorhaut (Präputium) teilweise oder vollständig durch einen chirurgischen Eingriff entfernt. ${ }^{25}$ Der substanzverletzende Eingriff in die körperliche Integrität erfüllt den objektiven Tatbestand der Körperverletzung in der Alternative des körperlichen Misshandelns ( $\$ 223$ Abs. 1 StGB). ${ }^{26}$ Er stellt zugleich eine Gesundheitsschädigung dar, da die vollständige Abheilung in der Regel ein bis zwei Wochen dauert. Für die Tatbestandsmäßigkeit der Zirkumzision kommt es nicht darauf an, ob der Eingriff medizinisch indiziert ist oder nicht, sofern man der Position der ständigen Rechtsprechung (Rechtfertigung durch Einwilligung) ${ }^{27}$ oder der im Vordringen befindlichen Ansicht der Literatur (Tatbe-

23 Joint United Nations Programme on HIV/AIDS (UNAIDS), Circumcision (Fn. 10), S. 7.

24 Joint United Nations Programme on HIV/AIDS (UNAIDS), Circumcision (Fn. 10), S. 7 unten.

25 In der Kinderchirurgie spricht man von kosmetischer (Präputium-sparender) und radikaler Zirkumzision (dazu M. Heinrich/K. K. Schäffer/D. von Schweinitz, Kinderchirurgie, Basiswissen und Praxis, München 2008, S. 189 f.; zu verschiedenen Operationstechniken R. Stein/J. W. Thüroff, Phimose, Der Urologe [B] 2002, S. 537 (539)).

26 Darüber hinaus kann die Qualifikation des $\$ 224$ Abs. 1 Nr. 2 StGB (Gefährliche Körperverletzung mittels eines gefährlichen Werkzeugs) erfüllt sein. Nach der Rspr. soll allerdings das von einem zugelassenen Arzt bestimmungsgemäß und kunstgerecht eingesetzte Skalpell aufgrund seiner konkret ungefährlichen Verwendung nicht unter $\$ 224$ Abs. 1 Nr. 2 StGB fallen (BGH NJW 1978, S. 1206; zustimmend H.-U. Paeffgen, in: U. Kindhäuser/U. Neumann/H.-U. Paeffgen (Hrsg.), Strafgesetzbuch,

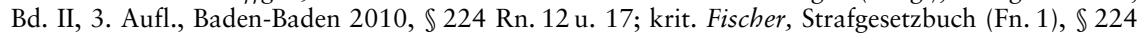
Rn. 9 a).

27 RGSt 25, 375 ff.; im Anschluss BGHSt 11, 111. 
standsausschluss durch Einwilligung $)^{28}$ folgt. ${ }^{29}$ Auf eine Darstellung des über 100jährigen Streits über die Tatbestandsmäßigkeit des ärztlichen Heileingriffs soll hier verzichtet werden, um Raum für weniger intensiv behandelte Fragen der rechtlichen Bedeutung der medizinischen Indikation zu gewinnen.

Der chirurgische Eingriff der Zirkumzision ist auch nicht als sozial adäquate Handlung tatbestandslos, wie dies in der Strafrechtswissenschaft teilweise vertreten wurde. ${ }^{30}$ Das unscharfe Kriterium der Sozialadäquanz muss sich nach der überzeugenden Auffassung von Roxin auf zwei Fallgruppen des Ausschlusses der objektiven Zurechnung zurückführen lassen. ${ }^{31}$ Die Beschneidung von Knaben stellt aber weder einen Fall des erlaubten Risikos dar, noch handelt es sich um eine geringfügige, sozial allgemein tolerierte Handlung, wobei es erneut nicht auf das Kriterium der medizinischen Indikation ankommt. Ein substanzverletzender chirurgischer Eingriff wird von der Rechtsordnung nicht wie bei der ordnungsgemäßen Verkehrsteilnahme ${ }^{32}$ von vorneherein und generell, sondern lediglich im Einzelfall gebilligt, sofern bestimmte weitere Voraussetzungen erfüllt sind, so dass ein genereller Ausschluss der objektiven Zurechnung unter dem Gesichtspunkt des erlaubten Risikos nicht in Betracht kommt. Eine geringfügige, sozial allgemein tolerierte Handlung liegt schon deshalb nicht vor, weil das Überschreiten der Erheblichkeitsschwelle bereits in der Tatbestandsdefinition des $\$ 223$ Abs. 1 StGB als ein „übles und unangemessenes Behandeln, das das körperliche Wohlempfinden nicht nur unerheblich beeinträchtigt" $" 33$ enthalten ist.

\section{Die Einwilligung des Minderjährigen}

Als Legitimationsquelle für die tatbestandliche Körperverletzung kommt zunächst die Einwilligung des Verletzten in Betracht, die je nach vertretener Auffassung tatbestandsausschließende oder rechtfertigende Wirkung hat. ${ }^{34}$

28 C. Roxin, Strafrecht Allgemeiner Teil, Grundlagen, Der Aufbau der Verbrechenslehre, 4. Aufl., München 2006, $\mathbb{S} 13$ Rn. 12 ff. und Rn. 26 mit weiteren Nachweisen.

29 A.A. nur die Tatbestandslösungen, grundlegend P. Bockelmann, Strafrecht des Arztes, Stuttgart 1968, S. 62 ff.; H. Lilie, in: B. Jähnke (Hrsg.), Strafgesetzbuch, Leipziger Kommentar, Bd. VI, 11. Aufl., Berlin 2005 , Vor $\$ 223$ Rn. 3 ff. mit weiteren Nachweisen.

30 T. Fischer, Strafgesetzbuch und Nebengesetze, 54. Aufl., München 2007, $\$ 223$ Rn. 6 b bezeichnete die Lösung über die Sozialadäquanz bis zur 54. Aufl. 2007 als „wohl hM“, versah dies aber bereits mit der Bemerkung: „zweifelhaft“. Inzwischen hat Fischer diese Auffassung aufgegeben, Fischer, Strafgesetzbuch (Fn. 1), $\$ 223$ Rn. 6 b.

31 Roxin, Strafrecht (Fn. 28), $\mathbb{S} 10$ Rn. 33 ff. (38-40).

32 Anerkannte Fallgruppen des erlaubten Risikos sind u.a. die Teilnahme am Schienen-, Straßen- oder Flugverkehr oder das Ausschenken von Alkohol in den gewerberechtlichen Grenzen.

33 Fischer, Strafgesetzbuch (Fn. 1), $\$ 223$ Rn. 3 a.

34 Zum Streitstand umfassend und mit weiteren Nachweisen Roxin, Strafrecht (Fn. 28), $\mathbb{1} 13$ Rn. $11 \mathrm{ff}$. sowie C. Roxin, Einwilligung, Persönlichkeitsautonomie und tatbestandliches Rechtsgut, in: M. Böse/ D. Sternberg-Lieben (Hrsg.), Grundlagen des Straf- und Strafverfahrensrechts, Festschrift für Knut Amelung zum 70. Geburtstag, Berlin 2009, S. 269 (271 ff.). 


\section{Objektive Schranken der Einwilligung in die Zirkumzision}

Die körperliche Integrität ist als Individualrechtsgut disponibel und für den Spezialfall der männlichen Beschneidung greifen auch keine objektiven Schranken der Einwilligung ein. Spezialgesetzliche Einwilligungssperren für Minderjährige, wie im Falle der Sterilisation (\$1631c S.2 BGB) und der Lebendorganspende ( $\int 8$ Abs. 1 S. 1 Nr. 1 a TPG), existieren für die Beschneidung nicht. ${ }^{35}$ Die männliche Beschneidung mit Einwilligung des Verletzten verstößt nach wohl allen vertretenen Deutungen der Sittenwidrigkeitsklausel des $\$ 228$ StGB zudem nicht gegen die guten Sitten. Verstoßen einverständliche Verletzungshandlungen nach Auffassung des BGH erst dann gegen die guten Sitten, „wenn bei vorausschauender objektiver Betrachtung aller maßgeblichen Umstände der Tat der Einwilligende in konkrete Todesgefahr gebracht wird“, 36 so stellt der Rechtsbegriff der guten Sitten jedenfalls für (wie auch immer motivierte) lege artis durchgeführte Zirkumzisionen von Knaben keine relevante Schranke der Einwilligung dar. ${ }^{37}$ Dasselbe gilt für die in der Literatur vorherrschenden eingeschränkten Schweretheorien zur Deutung des $\$ 228$ StGB. ${ }^{38}$ Für die Bestimmung der Grenzen der stellvertretenden Einwilligung bildet \$2 228 StGB demgegenüber schon keinen geeigneten Maßstab. ${ }^{39}$ Willigen die Sorgeberechtigten in einen im Sinne von $\mathbb{} 228$ StGB sittenwidrigen Eingriff beim Vertretenen ein, so stellt dies zwar stets zugleich einen Missbrauch ihrer Dispositionsbefugnis dar; die Grenzen der stellvertretenden Einwilligung müssen jedoch enger gezogen werden. ${ }^{40}$

Kaum mehr nachvollziehbar ist die im internationalrechtlichen Schrifttum vereinzelt anzutreffende Ansicht, die Beschneidung der männlichen Vorhaut sei, sofern sie nicht aus zwingenden medizinischen Gründen erfolge, stets als unmenschliche und erniedrigende Behandlung im Sinne von Art. 3 EMRK zu qualifizieren, selbst dann, wenn sie bei einwilligungsfähigen Minderjährigen und Erwachsenen mit deren Einwilligung erfolge. ${ }^{41}$ Sie verkennt die primär freiheitsrechtliche Funktion von Grund- und

35 Die Beschneidung von Knaben ist in der Regel auch nicht als körperliche Bestrafung, seelische Verletzung oder andere entwürdigende Erziehungsmaßnahme im Sinne von $\$ 1631$ Abs. 2 S. 2 BGB zu qualifizieren (dazu unten C.III.4.c)).

36 BGH NJW 2004, S. 2458 (2469) und nahezu wortgleich BGH NJW 2004, S. 1054 (1056 f.).

37 C. Jäger, Examens-Repetitorium Strafrecht Allgemeiner Teil, 4. Aufl., Heidelberg 2009, S. 114 mit 154. Die Genitalverstümmelung von Frauen kann dagegen schon aufgrund der mit ihr verbundenen

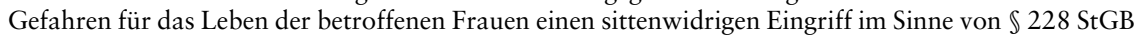
darstellen, der nicht durch Einwilligung gerechtfertigt werden kann (Fischer, Strafgesetzbuch (Fn. 1), \$ 223 Rn. 6 b)). Allg. zur Problematik der Genitalverstümmelung von Frauen D. Wüstenberg, Genitalverstümmelung und familienrechtliche Rechtsprechung, ZKJ 2008, S. 411.

38 H. J. Hirsch, in: B. Jähnke (Hrsg.), Strafgesetzbuch, Leipziger Kommentar, Bd. VI, 11. Aufl., Berlin 2005, \$228 Rn. 8 f.; Roxin, Strafrecht (Fn. 28), \$13 Rn. 41 unter Aufgabe seiner früheren Auffassung.

39 So aber wohl Jerouschek, Beschneidung (Fn. 3), S. 318.

40 Dazu unten III.4.

41 So ausdrücklich H. Gilbert, Time To Reconsider The Lawfulness Of Ritual Male Circumcision, European Human Rights Law Review 2007, S. 279 (291). 
Menschenrechten als Abwehrrechte des Einzelnen gegen den Staat. Als Fundamente personaler Autonomie ${ }^{42}$ sperren sie sich gegen Versuche, sie als hart paternalistische Eingriffsnormen des Staates zu instrumentalisieren. ${ }^{43}$

\section{Einwilligungsfähigkeit und medizinische Indikation}

Dispositionsberechtigt ist zunächst der Minderjährige selbst, die Wirksamkeit seiner Einwilligung in die Zirkumzision setzt aber weiter voraus, dass er einwilligungsfähig ist. Die Beurteilung der Einwilligungsfähigkeit richtet sich im Strafrecht nicht nach festen Altersgrenzen oder den Regeln, die für die zivilrechtliche Geschäftsfähigkeit gelten. ${ }^{44}$ Vielmehr kommt es darauf an, ob der Rechtsgutsträger über die natürliche Einsichts- und Urteilsfähigkeit verfügt, also nach seiner geistigen und sittlichen Reife imstande ist, Wesen, Bedeutung und Tragweite des konkreten Eingriffs zu erfassen und sein Verhalten danach auszurichten. In diesem Fall kommt es aus strafrechtlicher Sicht allein auf seine Entscheidung an. ${ }^{45}$ Nach den allgemeinen Grundsätzen des Medizinstrafrechts gelten Minderjährige unter 14 Jahren (Kinder) grundsätzlich als einwilligungsunfähig, während für die Altersstufe der 14-18-Jährigen (Jugendliche) stets eine Beurteilung der individuellen geistigen und sittlichen Reife im Hinblick auf den konkreten Eingriff erforderlich ist. ${ }^{46}$ Bei einfachen medizinischen Eingriffen kann die Einwilligungsfähigkeit in Einzelfällen schon bei 14-16-Jährigen vorliegen, bei 16-18-Jährigen ist dies zu vermuten. Für die Einwilligung Minderjähriger in die Zirkumzision gelten insoweit keine Besonderheiten: Weder folgt aus der Religionsmündigkeit ( $\$ 5$ RelKErzG), dass Minderjährige ab 14 Jahren stets in religiös motivierte körperliche Eingriffe einwilligen könnten, ${ }^{47}$ noch ergeben sich aus dem Fehlen

42 H. Dreier, Der freiheitliche Verfassungsstaat als riskante Ordnung, RW 2010, S. 11 (19f.).

43 T. Gutmann, Zur philosophischen Kritik des Rechtspaternalismus, in: U. Schroth/K. A. Schneewind/ T. Gutmann/B. Fateh-Moghadam (Hrsg.), Patientenautonomie am Beispiel der Lebendorganspende, Göttingen 2006, S. 189; zur Kritik des Strafrechtspaternalismus Roxin, Strafrecht (Fn. 28), $\mathbb{} 2$ Rn. 32; zu einer liberalen Interpretation der EMRK mit Blick auf die Beschneidung von Knaben Schiratzki, Body (Fn. 9), S. 8 ff.

44 K. Ulsenheimer, Arztstrafrecht in der Praxis, 4. Aufl., Heidelberg 2008, Rn. $108 \mathrm{ff}$.

45 Ulsenheimer, Arztstrafrecht (Fn. 44), Rn. 111; Roxin, Strafrecht (Fn. 28), $\$ 13$ Rn. 92. Die Entscheidung des OLG Hamm NJW 1998, S. 3424, wonach eine Minderjährige in jedem Fall, also auch dann, wenn sie einwilligungsfähig ist, der Zustimmung der Eltern zu einem Schwangerschaftsabbruch bedarf, ist daher im medizinstrafrechtlichen Schrifttum zu Recht auf Ablehnung gestoßen (Ulsenheimer, Arztstrafrecht (Fn. 44), Rn. 111 und 336). Zur Frage der Volljährigkeit als Voraussetzung der Einwilligungsfähigkeit bei ärztlichen Eingriffen, zu der Putzke (Putzke, Beschneidung, in: Putzke et al. (Hrsg.), Strafrecht (Fn. 3), S. 669 (685)) die Entscheidung des OLG Hamm heranziehen möchte, trifft die Entscheidung indes überhaupt keine Aussage.

46 U. Schroth, Die Einwilligung in eine nicht-indizierte Körperbeeinträchtigung zur Selbstverwirklichung - insbesondere die Einwilligung in Lebendspende, Schönheitsoperationen und Piercing, in: W. Hassemer/E. Kempf/S. Moccia (Hrsg.), In Dubio Pro Libertate, Festschrift für Klaus Volk zum 65. Geburtstag, München 2009, S. 719 (723 mit Fn. 22).

47 Zu weitgehend daher OLG Frankfurt, NJW 2007, S. 3580 (3581), das die Altersgrenzen des Gesetzes über die religiöse Kindererziehung unmittelbar für die Bestimmung der Einwilligungsfähigkeit in die Beschneidung heranzieht. Vgl. hierzu Putzke, Beschneidung, in: Putzke et al. (Hrsg.), Strafrecht (Fn. 3), S. 669 (684 f.) und Schroth, Einwilligung, in: Hassemer et al. (Hrsg.), FS Volk (Fn. 46), S. 719 (723 mit Fn. 22). 
einer medizinischen Indikation bei der Beschneidung eo ipso besonders strenge Anforderungen an die Einwilligungsfähigkeit. ${ }^{48}$ Die Religionsmündigkeit kann nicht allein maßgeblich sein, da das Gesetz über die religiöse Kindererziehung die Selbstbestimmung Minderjähriger bei der Wahl des Bekenntnisses schützt, die nicht mit der Verfügung über die körperliche Integrität vergleichbar ist. Umgekehrt fehlt es aber auch an einem unmittelbaren Zusammenhang zwischen medizinischer Indikation und Einwilligungsfähigkeit, weshalb es nicht überzeugen kann, wenn Teile der Literatur die Anforderungen an die Einwilligungsfähigkeit gerade bei nicht medizinisch indizierten Beschneidungen so hoch ansetzen wollen, dass sie stets ${ }^{49}$ oder zumindest in der Regel ${ }^{50}$ erst mit Volljährigkeit bejaht werden könne. Zunächst hat die medizinische Indikation ersichtlich keine Bedeutung für die Fähigkeit des Minderjährigen Art, Umfang, Folgen und Risiken des Eingriffs zu erfassen. Die Zirkumzision zählt nach allgemeiner Auffassung zu den einfachsten urologischen Eingriffen. Seine konkrete Durchführung und Folgen lassen sich anschaulich anhand von Fotos, Zeichnungen oder auch am eigenen Körper des Minderjährigen demonstrieren. Das Fehlen einer medizinischen Indikation könnte allenfalls dazu führen, dass die Bedeutung des Eingriffs schwerer zu erfassen ist. Der Minderjährige muss den Sinngehalt des Eingriffs und die Reichweite des Rechtsgutsverzichts derart erfassen können, dass er zu einer subjektiven Kosten-Nutzen-Abwägung des geplanten Opfers in der Lage ist. ${ }^{51}$ An dieser Stelle gewinnen die Wertungen des Gesetzes über die religiöse Kindererziehung ihre Bedeutung: Folgen aus $\ 5$ RelKErzG zwar keine festen Altersgrenzen für die Einwilligung in körperliche Eingriffe, so ist der Vorschrift doch negativ zu entnehmen, dass die Einsichtsfähigkeit eines religionsmündigen, also mindestens 14-Jährigen ${ }^{52}$ nicht mit der Begründung verneint werden kann, er sei nicht in der Lage die religiöse Bedeutung der Beschneidung zu verstehen. Die Anforderungen an die Selbstbestimmungsfähigkeit sind in religiösen Fragen nach der Wertung des Gesetzgebers eher niedrig anzusetzen. Das Konzept partieller Einwilligungsfähigkeit dient dabei gerade dem Schutz des Minderjährigen in besonders persönlichkeitsrelevanten Bereichen.

48 So aber offenbar Putzke, Beschneidung, in: Putzke et al. (Hrsg.), Strafrecht (Fn. 3), S. 669 (685 f.).

49 Jerouschek, Beschneidung (Fn. 3), S. 318.

50 Putzke, Beschneidung, in: Putzke et al. (Hrsg.), Strafrecht (Fn. 3), S. 669 (685 f.): in der Regel „(...) erst mit Erreichen der Volljährigkeit (...)“ (686); anders aber H. Putzke, Juristische Positionen zur religiösen Beschneidung, NJW 2008, S. 1568, (1570): „(...) in der Regel zwischen dem 16. Und 18. Lebensjahr (...)“.

51 K. Amelung, Vetorechte beschränkt Einwilligungsfähiger in Grenzbereichen medizinischer Intervention, Vortrag gehalten vor der Juristischen Gesellschaft zu Berlin am 22. Februar 1995, Berlin 1995, S. 10 .

$52 \$ 5$ RelKErzG. 
Der Versuch, dieses am Persönlichkeitsrecht Minderjähriger orientierte Schutzkonzept durch ein tutioristisches Integritätsschutzprinzip ${ }^{53}$ zu ersetzen, scheint maßgeblich auf der Überlegung zu beruhen, dass sich Minderjährige mit Blick auf die Frage der Zirkumzision dem „Einfluss von Familie, von Freunden und nicht zuletzt von der Gemeinschaft der Gläubigen “ in den wenigsten Fällen entziehen könnten. ${ }^{54}$ Diese Auffassung verkennt, dass der für die Persönlichkeitsbildung konstitutive Einfluss von Familien, Freunden und gegebenenfalls auch von Religionsgemeinschaften von der Rechtsordnung in der Regel nicht als problematisch, sondern als schützenswert betrachtet wird, wie sich an der grundrechtlichen Gewährleistung des Elternrechts in Art. 6 Abs. 2 GG und dem Schutz der religiösen Erziehung über Art. 4 Abs. 1 und 2 sowie 7 Abs. 2 GG und dem Gesetz über die religiöse Kindererziehung ablesen lässt. Diese äußeren Einflüsse werden erst dann rechtlich problematisch, wenn sie den Charakter einer strafrechtlichen Nötigung annehmen. ${ }^{55}$ Der Gesetzgeber kann das Risiko defizitärer Entscheidungen Minderjähriger in spezifischen Kontexten für so hoch bewerten, dass er ein gesetzliches Mindestalter festlegt und zugleich die Möglichkeit der Stellvertretung ausschließt. ${ }^{56}$ Ein solcher, auf der Logik des Verdachts gegen jüdische und muslimische Eltern beruhender, weich paternalistischer Gefährdungstatbestand liegt für den Fall der Zirkumzision von Knaben aber - aus guten Gründen - nicht vor.

\section{Vetorechte nicht einwilligungsfähiger Minderjähriger}

Dem Gedanken des Schutzes des Selbstbestimmungsrechts Minderjähriger verpflichtet ist dagegen die Anerkennung von ungeschriebenen Vetorechten, die bereits von nicht bzw. nur beschränkt einwilligungsfähigen Minderjährigen ausgeübt werden können. ${ }^{57}$ Es entspricht der herrschenden Auffassung im Medizinstrafrecht, dass einwilligungsfähige Minderjährige medizinische Eingriffe auch gegen den Willen ihrer Eltern ablehnen können. ${ }^{58}$ Das Konzept der Vetorechte geht darüber insofern hinaus, als die „Vetomündigkeit weiter reicht als die Einwilligungsmündigkeit““.59 Damit wird dem Gedanken Rechnung getragen, dass das Elternrecht mit zunehmen-

53 Der philosophische Begriff des Tutiorismus bezeichnet eine Haltung, die zwischen zwei Möglichkeiten immer die sicherere wählt.

54 So Putzke, Beschneidung, in: Putzke et al. (Hrsg.), Strafrecht (Fn. 3), S. 669 (685).

55 Dazu Schroth, Einwilligung, in: Hassemer et al. (Hrsg.), FS Volk (Fn. 46), S. 719 (728 ff.).

56 Vgl. etwa $\$ 8$ Abs. 1 S. 1 Nr. 1 a TPG; dazu B. Fateh-Moghadam, Die Einwilligung in die Lebendorganspende, Die Entfaltung des Paternalismusproblems im Horizont differenter Rechtsordnungen am Beispiel Deutschlands und Englands, München 2008, S. $241 \mathrm{ff}$. Zu den Grenzen solcher weich paternalistischen Regelungen vgl. B. Fateh-Moghadam, Grenzen des Paternalismus, Blinde Flecken der liberalen Paternalismuskritik, in: B. Fateh-Moghadam/S. Sellmaier/W. Vossenkuhl (Hrsg.), Grenzen des Paternalismus, Ulrich Schroth zum 60. Geburtstag, Stuttgart 2010, S. 21 (37 ff.).

57 Grundlegend Amelung, Vetorechte (Fn. 51).

58 Ulsenheimer, Arztstrafrecht (Fn. 44), Rn. 111.

59 K. Ulsenheimer, $\mathbb{1 3 9}$ Die fahrlässige Körperverletzung, in: A. Laufs (Hrsg.), Handbuch des Arztrechts, München 2002, S. 1235 (1250 Rn. 31); Ulsenheimer, Arztstrafrecht (Fn. 44), Rn. 110. 
der Selbstbestimmungsfähigkeit des Kindes zu weichen hat, wie dies unter anderem

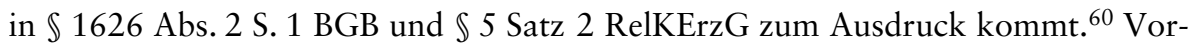
aussetzungen, Reichweite und Grenzen derartiger Vetorechte sind im Einzelnen ungeklärt. ${ }^{61}$ Gerade bei nicht oder nur relativ medizinisch indizierten Eingriffen mit der Möglichkeit erheblicher Folgen für die künftige Lebensgestaltung hat das Vetorecht Minderjähriger indes auch in der Rechtsprechung Anerkennung gefunden. ${ }^{62}$ Vetorechte kommen insbesondere dann in Betracht, wenn der Eingriff ohne gravierende gesundheitliche Folgen bis zur vollen Entscheidungskompetenz aufgeschoben werden kann. ${ }^{63}$ Der normative Sinn von Vetorechten aktualisiert sich mithin gerade in den Fällen nicht medizinisch zwingend gebotener Zirkumzisionen bei Knaben. Minderjährigen, die bereits über eine ausreichende natürliche Urteilsfähigkeit verfügen, steht in diesen Fällen ein Vetorecht gegen die Einwilligung der Personensorgeberechtigten zu. Bringen Sie ihre Ablehnung des Eingriffs erkennbar zum Ausdruck, so entfaltet die Einwilligung der Eltern auch im strafrechtlichen Sinne keine Wirkung. Der Arzt oder sonstige fachmännische Beschneidungspersonen müssen den Eingriff dann ablehnen.

\section{Stellvertretende Einwilligung in die Zirkumzision}

Praktische Bedeutung kommt der selbständigen Einwilligung einwilligungsfähiger Minderjähriger vor allem für Fälle (präventiv-)medizinisch, hygienisch oder ästhetisch motivierter Zirkumzisionen zu, da religiöse Beschneidungen häufig neonatal oder in einer frühkindlichen Phase durchgeführt werden. So erfolgt die Beschneidung von Knaben nach jüdischer Tradition am 8. Tag nach der Geburt, nach islamischer Tradition gibt es zwar kein zwingend vorgeschriebenes Alter, sehr häufig erfolgt die Beschneidung indes auch hier bereits neonatal und meistens bis zum Beginn der $\mathrm{Pu}$ bertät. ${ }^{64}$ In diesen Fällen können grundsätzlich die Personensorgeberechtigten stellvertretend in die Zirkumzision einwilligen, wobei weitgehend ungeklärt ist, wie die Grenzen der stellvertretenden Einwilligung zu bestimmen sind. Im Wege einer kritischen Rekonstruktion der strafrechtlichen Dogmatik der stellvertretenden Einwilligung wird im Folgenden ein spezifisch strafrechtlicher Maßstab für die Beurteilung der Grenzen der Dispositionsbefugnis bei der Einwilligung in die Beschneidung von Knaben entwickelt.

60 Vgl. auch Art. 12 Abs. 1 des Übereinkommens über die Rechte von Kindern (UN-Kinderrechtskonvention).

61 Kritisch C. Knauf, Mutmaßliche Einwilligung und Stellvertretung bei ärztlichen Eingriffen an Einwilligungsunfähigen, Zugleich ein Beitrag zur Patientenverfügung, Baden-Baden 2005, S. $109 \mathrm{ff}$.

62 BGH NJW 2007, S. 217; Ulsenheimer, Arztstrafrecht (Fn. 44), Rn.110; Coester (Fn.1), $\$ 1666$ Rn. 153; Paeffgen (Fn. 3), $\$ 228$ Rn. 16.

63 Ulsenheimer, Arztstrafrecht (Fn. 44), Rn. 110.

64 Joint United Nations Programme on HIV/AIDS (UNAIDS), Circumcision (Fn. 10), S. 3 f. 


\section{Die stellvertretende Einwilligung als Einwilligung des Verletzten}

Die Frage nach der strafrechtsdogmatischen Einordnung der stellvertretenden Einwilligung scheint sich zunächst leicht beantworten zu lassen: Die stellvertretende Einwilligung gilt als Einwilligung des Verletzten, wirkt also je nach vertretener Ansicht tatbestandsausschließend oder rechtfertigend. Sie ist im Rahmen der Voraussetzungen der Einwilligung bei der Frage der Dispositionsbefugnis zu prüfen. ${ }^{65}$ Fehlt dem Rechtsgutsträger die erforderliche Einsichtsfähigkeit, so können die Personensorgeberechtigen für ihn die Einwilligung erteilen. ${ }^{66}$ Die Verfügungsbefugnis des Rechtsgutsinhabers geht insoweit auf sie über. ${ }^{67}$ Zum Inhalt der Personensorge gehören insbesondere auch Einwilligungen in ärztliche Eingriffe. ${ }^{68}$ Bei Minderjährigen sind grundsätzlich die Eltern $(\mathbb{S} \mathbb{S} 1626,1627,1629,1631$ BGB) personensorgeberechtigt, wobei zu beachten ist, dass die stellvertretende Einwilligung in die Beschneidung in der Regel die Zustimmung beider Elternteile erfordert. ${ }^{69}$

Die strafrechtsdogmatische Gleichstellung von Einwilligung und stellvertretender Einwilligung verliert indes an Selbstverständlichkeit, wenn man nach dem inhaltlichen Grund für die legitimierende Wirkung der Einwilligung fragt. Mit der liberalen Einwilligungstheorie ist die Handlungsfreiheit des Einwilligenden als inhaltliche Basis der Einwilligung anzusehen: „Wenn Rechtsgüter der freien Entfaltung des Einzelnen dienen“, so die klassische Formulierung von Roxin, „kann keine Rechtsgutsverletzung vorliegen, wenn eine Handlung auf einer Disposition des Rechtsgutsträgers beruht, die seine freie Entfaltung nicht beeinträchtigt, sondern im Gegenteil deren Ausdruck ist. “ ${ }^{“ 70}$ Dass diese Begründung auch für Einwilligungssurrogate wie die stellvertretende Einwilligung trägt, scheint zumindest diskussionswürdig. Insoweit lassen sich zwei idealtypische Positionen unterscheiden. Die erste nimmt an, dass es sich bei der stellvertretenden Einwilligung um Fremdbestimmung handelt, ${ }^{71}$ bei welcher der Gedanke der Selbstbestimmung durch eine notstandsähnliche objektive In-

65 Vgl. nur das Prüfungsschema bei R. Rengier, Strafrecht, Allgemeiner Teil, München 2009, S. 217 f.

66 Roxin, Strafrecht (Fn. 28), $\mathbb{1 3}$ Rn. 92.

67 Rengier, Strafrecht (Fn. 65), S. 219.

68 U. Diederichsen, in: P. Bassenge/G. Brudermüller/U. Diederichsen/W. Edenhofer et al. (Hrsg.), Palandt, Bürgerliches Gesetzbuch mit Nebengesetzen, 68. Aufl., München 2009, \$1626 Rn. 10; B. Veit, in: H. G. Bamberger/H. Roth/Bamberger-Roth (Hrsg.), Kommentar zum Bürgerlichen Gesetzbuch, Internationales Privatrecht $\$ \mathbb{S} 1297$ - 2385, EGBGB, Bd. III, 2. Aufl., München 2008, $\$ 1626$ Rn. 23; Roxin, Strafrecht (Fn. 28), Rn. 92.

69 B.-R. Kern, Fremdbestimmung bei der Einwilligung in ärztliche Eingriffe, NJW 1994, S. 753 (756); KG Berlin FamRZ 2006, S. 142 (Impfung).

70 Roxin, Strafrecht (Fn. 28), \13 Rn. 12. Den liberalen Einwilligungsmodellen stehen kollektivistische Einwilligungsmodelle gegenüber, die auf dem Prinzip des überwiegenden Interesses beruhen, dazu $T$. Rönnau, Willensmängel bei der Einwilligung im Strafrecht, Tübingen 2001, S. 29-116 und FatehMoghadam, Einwilligung (Fn. 56), S. 90-108.

71 So ausdrücklich T. Rönnau, in: H. W. Laufhütte/R. Rissing-van Saan/K. Tiedemann (Hrsg.), Strafgesetzbuch, Leipziger Kommentar, Bd. II, 12. Aufl., Berlin 2006, Vor $\$ 32$ Rn. 180; Kern, Fremdbestimmung (Fn. 69). 
teressenabwägung, die medizinische Notwendigkeit ${ }^{72}$ oder durch die Prinzipien der mutmaßlichen Einwilligung zu ersetzen ist. ${ }^{73}$ Die zweite betrachtet das Selbstbestimmungsrecht des Verletzten als Grundlage von Einwilligung und stellvertretender Einwilligung. Im Falle der stellvertretenden Einwilligung überträgt die Rechtsordnung lediglich die Wabrnehmung des Selbstbestimmungsrechts auf die Personensorgeberechtigten. ${ }^{74}$ Reichweite und Grenzen des so konstruierten normativen Artefakts einer „Als-ob-Selbstbestimmung“, sind selbständig unter Berücksichtigung des besonderen Verhältnisses von Staat, Eltern und Kind zu bestimmen. Aus rechtsvergleichender Perspektive entsprechen die hier angesprochenen konstruktiven Alternativen der Gegenüberstellung der englischen Doktrin des besten Interesses (best interest) und dem deutschen Modell der stellvertretenden Einwilligung (substituted judgement).

\section{Best interest versus substituted judgement - rechtsvergleichende Überlegungen}

Für nicht einwilligungsfähige Erwachsene kannte das englische Recht bis zum Mental Capacity Act 2005 gar keine Stellvertretung, so dass für sie nach Notstandsgesichtspunkten (necessity) zu entscheiden war. ${ }^{75}$ Für Minderjährige sieht das englische Recht zwar grundsätzlich die Möglichkeit der stellvertretenden Einwilligung in medizinische Eingriffe vor. ${ }^{76}$ Die Eltern bleiben dabei aber nicht nur materiell streng an das objektiv zu bestimmende beste Interesse des Kindes gebunden, ihre Entscheidungsmacht wird vielmehr auch kompetenzmäßig dadurch begrenzt, dass die englischen Gerichte jederzeit an Stelle der Eltern über das beste Interesse jedes Kindes im Vaterland entscheiden können (parens patriae-jurisdiction). ${ }^{77}$ Bemerkenswerterweise lassen die englischen Gerichte in Ausübung ihrer Schutzfunktion medizinisch nicht indizierte Beschneidungen von Knaben in weitem Umfang zu. ${ }^{78}$ Der Grund hierfür besteht darin, dass das Kriterium des besten Interesses sich im Common Law traditionell nicht auf das medizinisch beste Interesse beschränkt, sondern insbesondere den subjektiv-kulturellen Kontext des Kindes berücksichtigt. So geht die Rechtsprechung implizit davon aus, dass die Beschneidung von Knaben im besten Interesse des

72 Kern, Fremdbestimmung (Fn. 69), S. 758 f.

73 Knauf, Einwilligungsunfähige (Fn. 61).

74 In diesem Sinne Roxin, Strafrecht (Fn. 28), $\$ 13$ Rn. 92 ff. und insb. Rn. 16: der Sorgeberechtigte übe eine Stellvertretung im Willen aus, so dass „die Einwilligung der Eltern in den rechtlich gezogenen Grenzen als die des Kindes gilt (...)“; zustimmend B. Tag, Der Körperverletzungstatbestand im Spannungsfeld zwischen Patientenautonomie und Lex artis, Eine arztstrafrechtliche Untersuchung, Berlin 2000, S. 74; ähnlich Rengier, Strafrecht (Fn. 65), S. 219.

75 I. Kennedy/A. Grubb, Principles of Medical Law, 2. Aufl., Oxford 2004, S. 254 f.; zu den grundlegenden Neuerungen durch den Mental Capacity Act vgl. Fateh-Moghadam, Einwilligung (Fn. 56), S. $207 \mathrm{ff}$.

76 Section 3 (1) Children Act 1989.

77 Kennedy/Grubb, Principles (Fn. 75), S. 206 ff.

78 Auch die Law Commission betont die Zulässigkeit ritueller Beschneidungen von Knaben, The Law Commission, Consent In The Criminal Law, A Consultation Paper, London 1995, S. $119 \mathrm{ff}$. 
Kindes ist, wenn sie dem Willen beider Elternteile entspricht. ${ }^{79}$ Bei Meinungsverschiedenheiten zwischen den Sorgeberechtigten liege eine religiöse oder rituelle Beschneidung dagegen nur dann im besten Interesse des Kindes, wenn es in einem stark religiös oder traditionell geprägten Kontext aufwachse. ${ }^{80}$

Der Vergleich mit dem englischen Recht ist deshalb interessant, weil auch das oben genannte Fremdbestimmungsmodell der stellvertretenden Einwilligung auf dem Prinzip des besten Interesses und der Vorstellung des Staates als parens patriae zu beruhen scheint. Das Prinzip des besten Interesses wird dabei über das Kriterium des Kindeswohls als Grenze der Dispositionsbefugnis der Eltern, gewissermaßen durch die Hintertür, in das deutsche Recht eingeführt. Anders als im englischen Recht soll das Kindeswohl bei körperlichen Eingriffen hingegen allein durch das medizinisch beste Interesse definiert werden. ${ }^{81}$ Die Beschneidung von Knaben entspricht dem Kindeswohl nach dieser Auffassung nur dann, wenn der Eingriff medizinisch zwingend notwendig ist. ${ }^{82}$ Die Legitimation der Zirkumzision ist nach dieser Auffassung durch eine objektive Abwägung ihrer (medizinischen) Vor- und Nachteile zu ermitteln, wobei die Vorteile die Nachteile überwiegen müssen. ${ }^{83}$ Hier tritt die Rechtsmacht als Erziehungskonkurrent neben die Eltern und entscheidet positiv, ob die Beschneidung objektiv im besten Interesse des Minderjährigen ist (Kindeswohl als positiver Standard). Den Eltern wird dabei kein Ermessen eingeräumt: Entweder liegt die medizinische Indikation vor oder nicht. Denkt man diesen Ansatz konsequent zu Ende, verliert die stellvertretende Einwilligung der Personensorgeberechtigten ihre eigenständige Bedeutung. So kommt eine neuere monographische Untersuchung zu dem Ergebnis, dass die Konstruktion einer Stellvertretung bei der Einwilligung für die Rechtfertigung ärztlicher Eingriffe an Einwilligungsunfähigen weder geeignet noch notwendig sei. ${ }^{84}$ Bei nicht einwilligungsfähigen Minderjährigen reduziere sich das Kindeswohl hinsichtlich körperlicher Eingriffe mangels elterlichem Interpretations-

79 (CA (Civ Div)) Court of Appeal (Civil Division), Re J (A Minor) (Specific Issue Orders: Muslim Upbringing and Circumcision), 25.11.1999; Family Division, Re J (A Minor) (Specific Issue Orders: Muslim Upbringing and Circumcision), 6.5.1999; Family Division, Re S (Children) (Specific Issue Order: Religion: Circumcision), 30.3.2004.

80 Family Division, Circumcision (Fn. 79); Family Division, Religion (Fn. 79).

81 Kern, Fremdbestimmung (Fn. 69), S. 758 f.; Rönnau (Fn. 71), Vor $\$ 32$ Rn. 180; Knauf, Einwilligungsunfähige (Fn. 61), S. 113 f. und zusammenfassend S. 169.

82 Putzke, Beschneidung, in: Putzke et al. (Hrsg.), Strafrecht (Fn. 3), S. 669 (707). Putzke scheint auch nicht medizinische Vor- und Nachteile der Beschneidung berücksichtigen zu wollen, lässt aber im Ergebnis nur kurativ-medizinisch zwingende Gründe gelten.

83 Putzke, Zirkumzision (Fn. 3); S. 270; Herzberg, Beschneidung (Fn. 3), S. 334 ff., der die Abwägung noch strikter auf objektive medizinische Vor- und Nachteile beschränkt. Hierdurch wird die Stellvertretung auch bei „medizinisch neutralen“ Eingriffen ausgeschlossen (Kern, Fremdbestimmung (Fn. 69), S. 758 f.).

84 Knauf, Einwilligungsunfähige (Fn. 61), zusammenfassend S. 169 und ausführlich S. 137 ff. Die Einwilligungsentscheidung des „trotzdem sogenannten Vertreters in Gesundheitsangelegenheiten“ sei lediglich als „indirektes Indiz für den mutmaßlichen Willen des Betroffenen“ einzuordnen. 
primat auf das Ergebnis einer objektiven Interessenabwägung und damit auf die medizinische Indikation. ${ }^{85}$

\section{Verfassungsrechtliche Garantie der stellvertretenden Einwilligung}

Die implizite oder explizite Ersetzung des Instituts der stellvertretenden Einwilligung im Medizinstrafrecht durch die Doktrin des medizinisch besten Interesses ist mit dem grundrechtlich gewährleisteten Vorrang der elterlichen Personensorge und seiner Konkretisierung im Familien- und Strafrecht nicht vereinbar. Das Verhältnis von elterlichem Sorgerecht und staatlichem Wächteramt in Art. 6 Abs. 2 GG schließt das Auftreten des Staates als parens patriae, d.h. als gleichberechtigtem Erziehungs- und Fürsorgekonkurrenten aus. Der Staat darf keine positive Entscheidung darüber treffen, was objektiv im besten Interesse des Kindes ist, sondern wird darauf beschränkt „die Kindeswohl-Bestimmung durch die Eltern einer Unvertretbarkeitskontrolle zu unterziehen. " ${ }^{86}$ Jestaedt betont zu Recht, dass es bei der Ausübung des staatlichen Wächteramts darum gehe, die Grenzen dessen (verbindlich) festzustellen, was unter keinem vertretbaren Gesichtspunkt mehr als dem Wohl des Kindes dienlich und damit als kindeswohlgefährdend oder als kindeswohlschädigend zu qualifizieren sei. ${ }^{87}$ Der Maßstab des Kindeswohls verweise hier auf einen „negativen Standard“. ${ }^{88}$ Damit liegt der strukturelle Unterschied zwischen der Doktrin des besten Interesses und dem im Grundgesetz garantierten Modell der stellvertretenden Einwilligung klar zu Tage: hier die positive Feststellung dessen, was in der konkreten Situation objektiv „das Beste“ für das Kind ist (Kindeswohl als positiver Standard); dort die negative Feststellung, ab wann die Ausübung der elterlichen Sorge (ausnahmsweise) unvertretbar ist (Kindeswohl als negativer Standard).

Mit der oben dargelegten verfassungsrechtlich gebotenen Umstellung des normativen Beurteilungsmaßstabes auf eine negative Bestimmung der Grenzen der elterlichen Sorge ist aber noch nicht beantwortet, unter welchen Voraussetzungen die stellvertretende Einwilligung in einen körperlichen Eingriff als unvertretbar zu qualifizieren ist. Es wäre immerhin denkbar, dies immer schon dann anzunehmen, wenn Eltern in einen körperlichen Eingriff einwilligen, der nicht zwingend medizinisch geboten ist, so dass die negative und die positive Bestimmung des Kindeswohls zum selben Ergebnis kämen. Für eine solche - im Ergebnis nicht überzeugende - restriktive Interpretation könnte prima facie die in der verfassungsrechtlichen Literatur zu Art. 6 Abs. 2 GG verbreitete Unterscheidung zwischen ausfüllungsbedürftigen Rechtspositionen (Religions- und Meinungsfreiheit; freie Entfaltung der Persönlich-

85 Knauf, Einwilligungsunfähige (Fn. 61), S. 169.

86 M. Jestaedt, in: R. Dolzer/C. Waldhoff/K. Graßhof (Hrsg.), Bonner Kommentar zum Grundgesetz, 139. Aufl., Heidelberg 2009, Art. 6 Abs. 2 und 3 Rn. 42 am Ende.

87 Jestaedt (Fn. 86), Art. 6 Abs. 2 und 3 Rn. 44.

88 Jestaedt (Fn. 86), Art. 6 Abs. 2 und 3 Rn. 44. 
keit) und objektivierbaren Rechtspositionen des Kindes (Menschenwürde, Recht auf Leben und körperliche Unversehrtheit) sprechen. ${ }^{89}$ Danach besteht für das elterliche Erziehungs- und Fürsorgeprimat dort kein Raum, wo das Kindeswohl nicht auf Ausfüllung durch die Eltern angewiesen ist, sondern objektiv bestimmt werden kann. Bei genauerem Hinsehen ist die Voraussetzung fehlender Ausfüllungsbedürftigkeit des Kindeswohls indes gerade in der Fallgruppe der stellvertretenden Einwilligung in medizinische und sonstige körperliche Eingriffe nicht erfüllt. Vollständig objektivierbar sind die genannten Grundrechtspositionen nämlich nur wenn und soweit sie „nicht eine bestimmte Freiheit der Betätigung, sondern einen Zustand schützen“. 90 Nur dann fehlt es an der Ausfüllungsbedürftigkeit der Grundrechtsposition, die immer dann entsteht, wenn die Grundrechtsverwirklichung eine gewisse Mündigkeit voraussetzt. Das durch Art. 2 Abs. 2 S. 1 GG garantierte und durch $\$ 223$ Abs. 1 StGB strafrechtlich abgesicherte Recht auf körperliche Unversehrtheit ist indes nicht auf einen objektiven Zustandsschutz gerichtet. Art. 2 Abs. 2 S. 1 GG gewährleistet vielmehr „zuvörderst Freiheitsschutz im Bereich der leiblich-seelischen Integrität" ${ }^{* 1}$ und auch $\$ 223$ Abs. 1 StGB schützt die körperliche Integrität nicht nur als eine „Ansammlung von Fleisch und Knochen“. ${ }^{92}$ Art. 2 Abs. 2 S. 1 GG und $\$ 223$ Abs. 1 StGB garantieren vielmehr ein körperbezogenes Selbstbestimmungsrecht, auf das es gerade bei der Einwilligung in medizinische Eingriffe ankommt: Bei Entscheidungen über die Vornahme von Heileingriffen, Impfungen, Schönheitsoperationen, das Stechen von Ohrlöchern, die Geschlechtszuweisung bei Intra-Sexualität und die Beschneidung von Knaben ist das Kindeswohl daher nur teilweise objektivierbar, teilweise aber auch ausfüllungsbedürftig. Die Wahrnehmung und Ausfüllung der körperbezogenen Selbstbestimmungsinteressen als grundrechtlich geschützter Teil der Personensorge obliegt vorrangig den Eltern. ${ }^{93}$ Eltern haben daher auch bei körperbezogenen Entscheidungen für ihre Kinder einen Ermessensspielraum, der erst dann überschritten ist, wenn sich die Entscheidung als evidenter Missbrauch des Sorgerechts darstellt. Dies schließt es aus, die Wirksamkeit körperbezogener Entscheidungen der Eltern allein an das Kriterium der medizinischen Indikation oder

89 Richtungsweisend Jestaedt (Fn. 86), Art. 6 Abs. 2 und 3 Rn. 134 ff. (136); dazu E. J. Lohse, Privatrecht als Grundrechtskoordinationsrecht - das Beispiel der elterlichen Sorge, Jura 2005, S. 815 (819); vgl. auch A. Schmitt-Kammler/C. von Coelln, in: M. Sachs (Hrsg.), Grundgesetz, Kommentar, 5. Aufl., München 2009, Art. 6 Rn. 59 ff. (61); mit etwas anderer Betonung R. Gröschner, in: H. Dreier (Hrsg.), Grundgesetz, Kommentar, Bd. I, 2. Aufl., Tübingen 2004, Art. 6 Rn. 109 („Umkehr der Begründungslast").

90 Jestaedt (Fn. 86), Art. 6 Abs. 2 und 3 Rn. 136.

91 BVerfGE 52, 131 (174); BVerfGE 89, 120 (130).

92 Roxin, Strafrecht (Fn. 28), $\mathbb{1 3}$ Rn. 14.

93 Auch Jestaedt unterwirft den ärztlichen Heileingriff nicht den Regeln für objektivierbare, sondern für ausfüllungsbedürftige Rechtspositionen des Kindes, da der ärztliche Eingriff auf Seite des Einwilligungsberechtigten Einwilligungsfähigkeit voraussetze, Jestaedt (Fn. 86), Art. 6 Abs. 2 und 3 Rn. 136; zustimmend Lohse, Privatrecht (Fn. 89), S. 819. Ganz dasselbe muss aber für die Einwilligung in medizinisch neutrale Eingriffe wie die Zirkumzision gelten. So i.E. auch Zähle, Religionsfreiheit (Fn. 6), S. $451 \mathrm{f}$. 
eine rein objektive Abwägung der Vor- und Nachteile des Eingriffs zu binden. Als Zwischenergebnis kann somit festgehalten werden, dass der These der Strafbarkeit der Beschneidung von Knaben mit dem Prinzip des besten medizinischen Interesses als Grenze der stellvertretenden Einwilligung ein rechtlich unzutreffender Beurteilungsmaßstab zugrunde liegt.

\section{Der Missbrauch der Dispositionsbefugnis als strafrechtliche Grenze der stellvertretenden Einwilligung}

Die strafrechtliche Grenze der Dispositionsbefugnis der Sorgeberechtigten muss die verfassungsrechtlichen Grundlagen des Verhältnisses von elterlicher Sorge und staatlichem Wächteramt berücksichtigen. Einen Orientierungspunkt bieten dabei

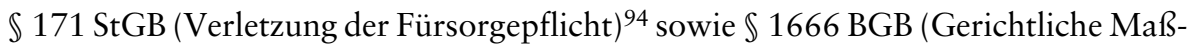
nahmen bei Gefährdung des Kindeswohls). ${ }^{95}$ Die genannten Vorschriften respektieren den grundsätzlichen Vorrang des Elternrechts bei der Bestimmung des Kindeswohls indem sie erst bei einer evidenten Überschreitung der Missbrauchsschwelle eingreifen. Nichts anderes muss für die Frage der strafrechtlichen Grenze der Dispositionsbefugnis bei der stellvertretenden Einwilligung in körperliche Eingriffe gelten, die daher als gröbliche Verletzung und damit als Missbrauch der elterlichen Sorgepflicht durch die eine Verletzung des Kindeswohls droht definiert werden kann. Eine in diesem Sinne missbräuchlich erteilte stellvertretende Einwilligung entfaltet auch strafrechtlich keine Wirksamkeit. ${ }^{96}$ Prüfungsmaßstab für die Feststellung einer gröblichen Verletzung und damit eines Missbrauchs der elterlichen Sorgepflicht hier liegt die entscheidende Differenz zu den am besten medizinischen Interesse orientierten Ansätzen - ist die Frage, ob das Ergebnis der primär von den Sorgeberechtigten vorzunehmenden Abwägung der Vor- und Nachteile des konsentierten Eingriffs unter keinem vertretbaren Gesichtspunkt noch mit dem Wohl des Kindes vereinbar ist. Diese Unvertretbarkeitskontrolle kann durch ein dreistufiges normatives Prüfungsprogramm konkretisiert und systematisiert werden. Die drei Prüfungsstufen betreffen dabei

94 Eine gröbliche Pflichtverletzung im Sinne des $\ 171$ StGB wird etwa im Fall der Genitalverstümmelung von Mädchen bejaht (Fischer, Strafgesetzbuch (Fn. 1), $\mathbb{1 7 1}$ Rn. 6).

95 Durch das Gesetz zur Erleichterung familiengerichtlicher Maßnahmen bei Gefährdung des Kindeswohls vom 4.7.2008 (KiWoMaG; BGBl I 1188) sollte keine Absenkung der durch den Gefährdungsbegriff bezeichneten Eingriffsschwelle bewirkt werden (Coester (Fn. 1), \$1666 Rn. 86). Zu den im Familienrecht anerkannten Fallgruppen für eine Kindeswohlgefährdung oder -verletzung gehören die auf Kindesmisshandlung beruhende Schädigung der körperlichen und seelischen Integrität (Coester (Fn. 1), \1666 Rn. 96) und zu Recht auch die drohende Genitalverstümmelung von Mädchen (Coester (Fn. 1), $\$ 1666$ Rn. 163).

96 Ähnlich Rengier, Strafrecht (Fn. 65), S. 219; aus zivilrechtlicher Sicht Diederichsen (Fn. 68), $\mathbb{S} 1626$ Rn. 12; BGH NJW 2003, S. 1824; BGH NJW 1954, S. 1576; anders Roxin, Strafrecht (Fn. 28), $\mathbb{S} 13$ Rn. 93, der einen Ausschluss der Stellvertretungsmöglichkeit bei „unvertretbaren Entscheidungen existentieller Art" annimmt; dies soll im Falle der stellvertretenden Einwilligung in eine Organspende $\mathrm{zu}$ bejahen, bei einer Blutspende zu verneinen sein. 
1. die Schwere des Eingriffs, seine gesundheitlichen Folgen und die mit ihm verbundenen Risiken bzw. (in Fällen der Einwilligungsverweigerung) die Schwere der Folgen und Risiken seiner Unterlassung;

2. die mit dem Eingriff verbundenen kurativ- und präventivmedizinischen Vorteile sowie sonstige Vorteile und Chancen;

3. das Fehlen von spezifisch kindeswohlverletzenden Eingriffsmodalitäten, insbesondere körperliche Bestrafungen, seelische Verletzungen, Demütigungen, Diskriminierung und andere entwürdigende Maßnahmen.

Die auf den Stufen 1 und 2 ermittelten Vor- und Nachteile des körperlichen Eingriffs sind - mit Blick auf die Vertretbarkeit der Elternentscheidung - gegeneinander abzuwägen, während das Vorliegen von spezifisch kindeswohlverletzenden Eingriffsmodalitäten (Stufe 3) eine absolute Einwilligungssperre bildet. Der so konkretisierte Prüfungsmaßstab für den Missbrauch des Sorgerechts präjudiziert nicht für alle Fälle eindeutige Ergebnisse, er ermöglicht aber eine differenzierte Beurteilung im Einzelfall und kann somit der Vielfalt ärztlicher Eingriffe bei Minderjährigen vom Ohrlochstechen über Schönheitsoperationen bis zur Beschneidung von Knaben oder gar der Genitalverstümmelung bei Mädchen gerecht werden. Unterschiede in der rechtlichen Behandlung bleiben hier als Ergebnis normativ-juristischer Wertungen transparent, anstatt über das medizinisch-deskriptive Kriterium der Indikation verschleiert zu werden. Auf den Fall der Beschneidung von Knaben angewendet ergibt sich daraus folgendes Bild:

\section{a) Die Schwere des Eingriffs, seine gesundheitlichen Folgen und Risiken}

Die Zirkumzision von Knaben stellt einen relativ leichten Eingriff in die Körperintegrität dar. Sie führt zwar zum irreversiblen Verlust der Vorhaut, hat aber bei fachgerechter Durchführung keine negativen gesundheitlichen Folgen für den Minderjährigen. ${ }^{97}$ Die Behauptung einer Beeinträchtigung des Sexuallebens beschnittener Männer aufgrund des Verlustes empfindungsfähiger Bereiche der Vorhaut entbehrt sexualmedizinischer Evidenz und dürfte eher als Ausdruck eines Ressentiments zu deuten sein. ${ }^{98}$ Das Komplikationsrisiko bei der Beschneidung von Knaben ist gering,

97 Bei der Genitalverstümmelung von Mädchen handelt es sich dagegen um einen gravierenden Eingriff, der mit lebensbedrohlichen Risiken verbunden ist und zu extensiven und lebenslangen Gesundheitsproblemen führt. Schwere und andauernde (Langzeit-)Komplikationen der weiblichen Genitalverstümmelung sind Schmerzen, Schock, Blutungen, Tetanus, Sepsis, Urinstau und Geschwüre. Weitere direkte Folge ist die Reduktion oder Verhinderung eines normalen Sexuallebens (Deprivation) sowie ein erhöhtes Komplikations- und Mortalitätsrisiko für Kind und Mutter bei der Geburt (zum Ganzen Wüstenberg, Rechtsprechung (Fn. 37) sowie Zähle, Religionsfreiheit (Fn. 6), S. 443 f und 446).

98 B. J. Morris, Why circumcision is a biomedical imperative for the 21st century, BioEssays 2007, S. 1147 (1154) mit weiteren Nachweisen. In der Sexualmedizin gibt es, obwohl weltweit $33 \%$ aller Männer über 15 Jahren beschnitten sind, keine Erkenntnisse darüber, dass sich die Zirkumzision nachteilig auf das Sexualleben beschnittener Männer auswirkt. Aus dem Umstand, dass die Entfernung der Vorhaut möglicherweise physiologische Auswirkungen auf die Empfindungsfähigkeit der Eichel hat (dazu Putzke, Beschneidung, in: Putzke et al. (Hrsg.), Strafrecht (Fn. 3), S. 669 (677)), folgt noch kein sexualmedizinisch nachteiliger Befund. 
sofern die Beschneidung lege artis in klinischen Settings durchgeführt wird. Das Risiko von Komplikationen wie Nachblutungen, Schmerzen und Entzündungen, die in der Regel leicht und vorübergehend sind, wird nach amerikanischen und israelischen Studien mit 0,2-0,4 \%, bei Einbeziehung minimaler Risiken bis $2 \%$ beziffert. ${ }^{99} \mathrm{Zum}$ lege-artis-Standard gehört nach dem gegenwärtigen Stand der medizinischen Wissenschaft eine angemessene Anästhesie zur Vermeidung von operativen und postoperativen Schmerzen und zwar auch bei Neugeborenen. ${ }^{100}$ Wird die Zirkumzision außerhalb klinischer Settings durchgeführt, können die Komplikationsraten und die Schwere der Komplikationen erheblich steigen, dies hängt aber maßgeblich von den konkreten Umständen ab. So können speziell geschulte und erfahrene jüdische oder muslimische Beschneider sehr gute Ergebnisse erzielen, während es bei traditionellen Beschneidungen, die in äußerst einfachen Verhältnissen im südlichen Afrika durchgeführt werden, zu schweren Komplikationen kommen kann. ${ }^{101}$

\section{b) Kurativ- und präventivmedizinische Vorteile sowie sonstige Vorteile und Chancen}

Die Zirkumzision von Knaben ist mit einer Reihe von medizinischen und hygienischen Vorteilen verbunden, die vor allem in den USA zu einer anhaltenden Diskussion darüber geführt haben, ob die neonatale Zirkumzision als Routinemaßnahme empfohlen werden sollte. ${ }^{102}$

Bei der kurativ-medizinisch indizierten Zirkumzision dient die Beschneidung der Beseitigung eines pathologischen Zustandes. Die häufigste Indikation für die Beschneidung ist dabei eine pathologische Verengung der Vorhaut (Phimose), die ein $\mathrm{Zu}$ rückstreifen über die Glans verhindert. ${ }^{103}$ Die Phimose führt zu Problemen beim Wasserlassen (Ballonierung, Schmerzen, Nachtröpfeln), kann chronische oder wiederkehrende Entzündungen hervorrufen und ab der Pubertät zu Schmerzen bei der Erektion und Problemen beim Geschlechtsverkehr einschließlich damit verbundener psychologischer Probleme führen. Insgesamt müssen etwa 2-4 \% aller Knaben und Männer im Laufe ihres Lebens aufgrund einer Phimose, Paraphimose oder rezidivierenden Entzündungen (Balanoposthitiden) zirkumzidiert werden. ${ }^{104}$

99 Joint United Nations Programme on HIV/AIDS (UNAIDS), Circumcision (Fn. 10), S. 17 f.: „Neonatal male circumcision is a relatively simple, quick and safe procedure when performed in a clinical setting under aseptic conditions by trained professionals. Complications rates are between 1 in 500 and 2 in 100 and are usually minor.".

100 Vgl. nur American Academy of Pediatrics, Circumcision Policy Statement, Pediatrics 1999, S. 686 (688 und 691).

$101 \mathrm{Zu}$ den Einzelheiten Joint United Nations Programme on HIV/AIDS (UNAIDS), Circumcision (Fn. 10), S. 19 ff.

102 Die Genitalverstümmelung von Mädchen ist dagegen mit keinerlei (präventiv-)medizinischen oder sonstigen Vorteilen verbunden.

103 Ein enges Präputium ist zum Zeitpunkt der Geburt und in den ersten Lebensjahren physiologisch (normal), so dass die Phimose keine Indikation für eine neonatale Zirkumzision darstellt (Heinrich/ Schäffer/von Schweinitz, Kinderchirurgie (Fn. 25), S. 189).

104 Heinrich/Schäffer/von Schweinitz, Kinderchirurgie (Fn. 25), S. 190. 
Als alternative Behandlungsmethode kommt bei einer Vorhautverengung die Behandlung mit cortisonhaltigen Cremes, die über einen Zeitraum von 4-6 Wochen mehrmals täglich auf die Vorhaut aufgetragen werden, in Betracht. ${ }^{105}$ Die Abwägung zwischen Behandlungsalternativen, wie vorliegend zwischen der Zirkumzision als sicherer und effektiver Behandlungsmethode und einer längerfristigen konservativen Behandlung mit unsicherem Behandlungserfolg, ist ein exemplarischer Fall für die Ausfüllungsbedürftigkeit der körperbezogenen Selbstbestimmungsinteressen des Minderjährigen. Diese Ausfüllung obliegt den Personensorgeberechtigten und nicht Ärzten oder Juristen. ${ }^{106}$ Den Eltern steht daher ein Wahlrecht zwischen beiden Behandlungsalternativen zu. Ein Missbrauch des Sorgerechts kommt in dieser Fallgruppe nur dann in Betracht, wenn die Eltern - etwa radikale Beschneidungsgegner - eine medizinisch notwendige Zirkumzision verweigern. ${ }^{107}$

Die Beschneidung ist aber auch dann mit nicht unerheblichen gesundheitlichen, insbesondere präventiv-medizinischen und hygienischen Vorteilen verbunden, wenn sie nicht der unmittelbaren Behebung von Krankheiten dient. Die WHO spricht insoweit von präventiven Indikationen für die Beschneidung von Knaben. ${ }^{108}$ Nachgewiesene Hygiene-Vorteile der Beschneidung bilden eine wichtige Determinante für nicht-religiöse Beschneidungen in den USA, aber etwa auch im südlichen Afrika. ${ }^{109}$ Der Umstand, dass die Intimhygiene bei Knaben auch ohne Zirkumzision gewährleistet werden kann, ändert nichts daran, dass der positive Zusammenhang zwischen Beschneidung und Intimhygiene ein Gesichtspunkt ist, der für die Frage der Vertretbarkeit der Entscheidung der Sorgeberechtigten von Bedeutung ist. Darüber hinaus bestehen nach den Erkenntnissen evidenzbasierter Medizin schlüssige Beweise dafür, dass beschnittene Männer ein signifikant niedrigeres Risiko haben an Harnwegsinfektionen, Peniskrebs, Syphilis und weichem Schanker (Chancroid) zu erkranken sowie sich durch hetero-sexuelle Sexualkontakte mit HIV zu infizieren. ${ }^{110}$ Zudem vermindert die Beschneidung die Übertragung von humanen Papillomaviren und senkt damit das Risiko von Gebärmutterhalskrebs bei den weiblichen Sexualpartnern beschnit-

105 Vgl. Heinrich/Schäffer/von Schweinitz, Kinderchirurgie (Fn.25), S. 190 sowie Stehr/Putzke/Dietz, Zirkumzision (Fn. 4), S. 1779.

106 A.A. offenbar Stehr/Putzke/Dietz, Zirkumzision (Fn. 4), S. 1779.

107 Zur Fallgruppe der Verweigerung medizinisch notwendiger Eingriffe Roxin, Strafrecht (Fn. 28), $\mathbb{1 3}$ Rn. 92 und Coester (Fn. 1), $\$ 1666$ Rn. 102.

108 Joint United Nations Programme on HIV/AIDS (UNAIDS), Circumcision (Fn. 10), S. 15.

109 Joint United Nations Programme on HIV/AIDS (UNAIDS), Circumcision (Fn. 10), S. 13 und S. 7 mit empirischen Daten.

110 Joint United Nations Programme on HIV/AIDS (UNAIDS), Circumcision (Fn. 10), S. 15 ff. mit weiteren Nachweisen. Zudem entfällt das Risiko der Entwicklung einer Phimose, die häufig nicht rechtzeitig erkannt wird und daher mit erheblichen körperlichen und psychischen Belastungen bei Heranwachsenden verbunden sein kann. 
tener Männer. ${ }^{111}$ Aufgrund der nachgewiesenen Reduzierung des Infektionsrisikos bei HIV um 48-60 \%, unter anderem durch drei randomisiert kontrollierte Studien in Südafrika, Uganda und Kenia, haben WHO und UNAIDS umfangreiche Beschneidungsprogramme im südlichen Afrika initiiert. ${ }^{112}$

Für den vorliegenden Zusammenhang ist nicht entscheidend, ob die Präventionsvorteile der Zirkumzision ihre Durchführung als neonatale Routinemaßnahme auch in Regionen mit anderen epidemiologischen Bedingungen rechtfertigen, wie dies gegenwärtig in den USA diskutiert wird. ${ }^{113}$ Vielmehr folgt aus den präventiv-medizinischen Vorteilen unmittelbar, dass die individuelle Entscheidung der Sorgeberechtigten für die Zirkumzision nicht als Sorgerechtsmissbrauch qualifiziert werden kann, der unter keinem vertretbaren Gesichtspunkt mit dem Kindeswohl vereinbar ist. ${ }^{114}$ Die American Academy of Paediatrics sieht diesen Unterschied in erfreulicher Klarheit:

„Existing scientific evidence demonstrates potential medical benefits of newborn male circumcision; however, these data are not sufficient to recommend routine neonatal circumcision. In the case of circumcision, in which there are potential benefits and risks, yet the procedure is not essential to the child's

111 X. Castellsagué/X. Bosch/N. Munoz/C. Meijer et al., Male Circumcision, Penile Human Papillomavirus Infection, And Cervical Cancer In Female Partners, The New England Journal of Medicine 2002, S. 1105; A. A. R. Tobian/D. Serwadda/T. Quinn/G. Kigozi et al., Male Circumcision for the Prevention of HSV-2 and HPV Infections and Syphilis, The New England Journal of Medicine 2009, S. 1298.

112 B. Auvert/D. Taljaard/E. e. a. Lagarde, Randomized, controlled intervention trial of male circumcision for reduction of HIV infection risk: the ANRS 1265 Trial, Public Library of Science Medicine 2005, S. 1112; R. H. Gray/G. Kigozi/D. Serwadda/F. Makumbi et al., Male circumcision for HIV prevention in men in Rakai, Uganda: a randomised trial, The Lancet 2007, S. 657; R. C. Bailey/ S. Moses/C. B. Parker/K. Agott et al., Male circumcision for HIV prevention in young men in Kismu, Kenya: a randomised controlled trial, The Lancet 2007, S. 643. Die Ergebnisse wurden durch mehrere systematische Reviews bestätigt, vgl. etwa Siegfried/Muller/Volmick/Deeks et al., Male circumcision for the prevention of heterosexual acquisition of HIV in men, Cochrane Database of Systematic Reviews 2003, Issue 3, http://www.cochrane.org/reviews/en/ab003362.html, Stand vom: 5.2.2010; zum Ganzen auch Joint United Nations Programme on HIV/AIDS (UNAIDS), Circumcision (Fn. 10), S. 13 ff. und 23 ff.; Centers For Disease Control And Prevention, CDC HIV/AIDS Science Facts: Male Circumcision and Risk for HIV Transmission and Other Health Conditions: Implications for the United States und Morris, Circumcision (Fn. 98), S. $1147 \mathrm{ff}$.

113 J. D. Dickerman, Circumcision in the Time of HIV: When Is There Enough Evidence to Revise the American Academy of Pediatrics' Policy on Circumcision?, Pediatrics 2007, S. 1006; P. S. Sullivan/ P. H. Kilmarx/T. A. Peterman/A. W. Taylor et al., Male Circumcision for the Prevention of HIV Transmission: What the New Data Mean for HIV Prevention in the United States, Public Library of Science Medicine 2007, S. 1162; Morris, Circumcision (Fn. 98); A. A. R. Tobian/R. H. Gray/T. C. Quinn, Male Circumcision for the Prevention of Acquisition and Transmission of Sexually Transmitted Infections, The Case for Neonatal Circumcision, Archieves of Pediatrics \& Adolescent Medicine 2010, S. 78; M. T. Brady, Newborn Circumcision, Routine or Not Routine, That Is the Question, Archieves of Pediatrics \& Adolescent Medicine 2010, S. 94.

114 So auch J. Laurance, Certainty on circumcision?, The Lancet 2009, S. 2017; M. A. Moreno, Male Circumcision, New Information About Health Benefits, Archieves of Pediatrics \& Adolescent Medicine 2010, S. 104; Brady, Circumcision (Fn. 113), S. 95. 
current well-being, parents should determine what is in the best interest of the child." $" 115$

\section{c) Das Fehlen von spezifisch kindeswohlverletzenden Eingriffsmodalitäten}

Die Zirkumzision von Knaben ist in der Regel nicht mit spezifisch kindeswohlverletzenden Eingriffsmodalitäten verbunden. Indizierte wie nicht indizierte Zirkumzisionen erfolgen nicht zum Zwecke der körperlichen Bestrafung und haben auch keinen demütigenden, diskriminierenden oder sonst entwürdigenden Charakter. ${ }^{116} \mathrm{Ei}$ nen demütigenden und entwürdigenden Charakter kann die Zirkumzision von Knaben indes insbesondere dann annehmen, wenn Vetorechte der Betroffenen nicht beachtet werden. Die mit der Praxis jüdischer oder muslimischer Beschneidungen verbundene religiös-zeremonielle Einrahmung und religionsinterne Bedeutung der Beschneidung stellt dagegen keine kindeswohlverletzende Modalität dar. Entscheidungen über die Religionszugehörigkeit und die religiöse Kindererziehung sind von der elterlichen Sorge umfasst und genießen über Art. 4 GG zusätzlichen Schutz. Dass sich Eltern bei der Entscheidung zur Zirkumzision auch oder ausschließlich von religiösen, kulturellen und traditionellen Erwägungen leiten lassen, ist daher rechtlich unproblematisch.

\section{d) Ergebnis}

Die Entscheidung der Eltern für die Zirkumzision von Knaben stellt in der Regel keinen Missbrauch der elterlichen Sorge dar. Dies steht für die kurativ-medizinischen Indikationen, auch bei bestehenden Behandlungsalternativen, außer Frage. Aber auch nicht kurativ-medizinisch indizierte Zirkumzisionen stellen grundsätzlich vertretbare Elternentscheidungen dar. Insoweit stehen einem relativ geringfügigen körperlichen Eingriff, der nicht mit negativen gesundheitlichen Folgen und mit einem nur geringen Risiko leichter Komplikationen verbunden ist, erhebliche präventivmedizinische Vorteile gegenüber. Wird in der internationalen Public Health Diskussion sogar darüber diskutiert, die Beschneidung von Knaben als neonatale Routinemaßnahme zu empfehlen, kann die individuelle Entscheidung der Eltern für die Zirkumzision nicht als Missbrauch des elterlichen Sorgerechts qualifiziert werden. Dabei kommt es nicht darauf an, ob sich die Sorgeberechtigen bei ihrer Entscheidung von diesen präventiv-medizinischen Erwägungen oder von religiösen oder traditionellen Erwägungen leiten lassen. Nur eine objektiv missbräuchliche Ausübung des Personensorgerechts kann zur Unwirksamkeit der stellvertretenden Einwilligung in

115 American Academy of Pediatrics, Circumcision (Fn. 100), S. 691. Das Policy Statement aus dem Jahr 1999 wurde 2005 bestätigt und wird gegenwärtig aufgrund der neuen präventiv-medizinischen Erkenntnisse erneut überdacht.

116 Hierin liegt ein weiterer wesentlicher Unterschied zur Genitalverstümmelung bei Mädchen und Frauen, die stets einen demütigenden und entwürdigenden Charakter hat und eine Manifestation der Diskriminierung von Frauen darstellt (vgl. bereits oben Fn. 97 und 102 sowie Zäble, Religionsfreiheit (Fn. 6), S. 447). 
die Zirkumzision führen. Ein Missbrauch ist nur dann anzunehmen, wenn die Zirkumzision nicht lege artis durchgeführt werden soll, Vetorechte Minderjähriger missachtet werden oder sonst entwürdigende Eingriffsmodalitäten vorliegen.

\section{Religionsfreiheit und Zirkumzision}

Da nach dem hier vertretenen Ansatz auch religiöse Beschneidungen regelmäßig durch die stellvertretende Einwilligung der Sorgeberechtigten gerechtfertigt werden können, liegt die Bedeutung des Grundrechts der Religionsfreiheit, neben ihrer Ausstrahlungswirkung auf die Bestimmung des Inhalts der elterlichen Sorge, vor allem in ihrer Abwehrdimension.

\section{Religiöse Rechtfertigung?}

Die Legitimation auch religiöser Beschneidungen folgt nicht aus einem partikularen Erlaubnissatz, der (nur) den Anhängern bestimmter Glaubensrichtungen die Überschreitung sonst allgemein geltender rechtlicher Grenzen gestatten würde, sondern aus der allgemeinen Bestimmung der Reichweite und Grenzen des elterlichen Sorgerechts. Es handelt es sich also gerade nicht um eine „religiöse Begründung von Normen “. ${ }^{117}$ Auch das säkulare amerikanische Elternpaar darf sich aus kulturellen, traditionellen, ästhetischen oder präventiv-medizinischen Gründen für die Beschneidung ihres Sohnes entscheiden. Daher kann an dieser Stelle offengelassen werden, ob die Religionsfreiheit überhaupt einen selbständigen strafrechtlichen Rechtfertigungsgrund bildet, wie dies für die Kunstfreiheit angenommen wird. ${ }^{118}$ Die speziellere Bestimmung der Grenzen der elterlichen Sorge, wie sie oben unter III. vorgenommen wurde, präjudiziert das Ergebnis der Abwägung zwischen Religionsausübungsfreiheit und den über das Kindeswohl geschützten eigenen Grundrechtspositionen des Kindes. Ist die Einwilligung in die Zirkumzision (ausnahmsweise) als Missbrauch des Sorgerechts und damit als Verletzung des Kindeswohls zu qualifizieren, weil sie nicht lege artis, unter Missachtung von Vetorechten oder unter sonst entwürdigenden Umständen erfolgt, so entfaltet die Einwilligung der Eltern keine Wirksamkeit. Eine religiöse Rechtfertigung für die Verletzung des Kindeswohls ist schlechthin ausgeschlossen. Darüber hinaus kommt bei religiös motivierten Verletzungen von Individualrechtsgütern Dritter regelmäßig auch auf Schuldebene ein Ausschluss der strafrechtlichen Verantwortung unter dem Gesichtspunkt der ebenfalls auf Art. 4 GG gestützten Gewissenstat nicht in Betracht. ${ }^{119}$

117 Missverständlich Schwarz, Beschneidung (Fn. 6), S. 1129. Auch dort wo das Recht einen Freiraum für religiös motivierte Sorgerechtsentscheidungen der Eltern - bis zur Grenze des Missbrauchs des Sorgerechts - eröffnet, ist dies nicht religiös begründet: der rechtlichen Zulässigkeit der männlichen Beschneidung liegt die Systemreferenz des Rechts und nicht die der Religion zugrunde.

118 Zur Freiheit der Kunst als eigenständigem Rechtfertigungsgrund Roxin, Strafrecht (Fn. 28), $\mathbb{1} 18$ Rn. 49 ff.

119 Roxin, Strafrecht (Fn. 28), \$ 22 Rn. 115. 


\section{Religionsfreiheit als Abwehrrecht}

Die Bedeutung der Religionsfreiheit als Abwehrrecht gegen unverhältnismäßige Beschränkungen der Beschneidung von Knaben aus religiösen Gründen bleibt hiervon unberührt. Soweit rechtliche Beschränkungen der Zirkumzision auch religiös motivierte Beschneidungen betreffen greifen sie in die Religionsausübungsfreiheit (forum externum) ein und müssen daher nicht nur im Hinblick auf das grundrechtlich geschützte Elternrecht aus Art. 6 Abs. 2 GG, sondern auch im Lichte von Art. 4 Abs. 1 und 2 GG verhältnismäßig sein. ${ }^{120}$ Für die Eröffnung der Religionsausübungsfreiheit genügt es dabei, dass die Zirkumzision religiös motiviert ist, sie muss nicht zwingend von der jeweiligen Religion vorgeschrieben sein. ${ }^{121} \mathrm{Ob}$ ein generelles Verbot der religiös motivierten Beschneidung von Knaben verfassungsrechtlich gerechtfertigt werden könnte, ist zweifelhaft. ${ }^{122}$ Für ein strafrechtliches Verbot der Zirkumzision von Knaben, das sich international als gesundheits-, kriminal- und integrationspolitischer Sonderweg darstellen würde, besteht nach den bisherigen Feststellungen auch kein rechtspolitisches Bedürfnis. Mit Blick auf den Schutz des Kindeswohls erscheinen nur solche Maßnahmen als legitim und zweckmäßig, die auf die medizinische Qualitätssicherung und die Beachtung von Vetorechten Minderjähriger gerichtet sind. ${ }^{123}$ Die Schwierigkeiten bei der Implementation des schwedischen Gesetzes zur Beschneidung von Knaben ${ }^{124}$ zeigen indes, dass der Einsatz des Strafrechts auch hierfür nicht unbedingt ein geeignetes Mittel ist. Nach der schwedischen Regelung, die strafrechtlich abgesichert ist, dürfen Knaben nur durch einen zugelassen Arzt oder - bei Säuglingen bis zu 2 Monaten - durch eine von der nationalen Gesundheitsbehörde zertifizierte Person (z.B. einen jüdischen Mohel) in Anwesenheit eines zugelassenen Arztes beschnitten werden. ${ }^{125}$ Ungeachtet dessen werden bei mehr als zwei Drittel der ca. 3000 muslimischen Beschneidungen, die jährlich in Schweden durchgeführt werden, die gesetzlichen Vorgaben nicht beachtet. ${ }^{126}$ Ursächlich hierfür scheinen vor allem Informationsdefizite und negative Erfahrungen

120 Schwarz, Beschneidung (Fn. 6), S. 1126 f.; für die männliche Beschneidung bejaht auch Zähle die Eröffnung des Schutzbereichs der Religionsausübungsfreiheit, während er dies für die weibliche Genitalverstümmelung unter Berufung auf den ordre public verneint (Zähle, Religionsfreiheit (Fn. 6), S. 443).

121 Zum weiten Schutzbereich der Religionsausübungsfreiheit vgl. BVerfGE 32, 98 (106).

122 Zur Verletzung von Art. 9 EMRK durch ein Totalverbot der Beschneidung vgl. Schiratzki, Body (Fn. 9).

123 Vgl. aus ethischer Perspektive M. Brusa/M. Y. Barilan, Cultural Circumcision in EU Public Hospitals - An ethical discussion, Bioethics 2009, S. 470.

124 Sw. Lag (2001:499) om omskärelse.

125 Der Arzt ist insbesondere für die Anästhesie zuständig. Darüber hinaus verlangt das Gesetz ausdrücklich die Beachtung von Vetorechten Minderjähriger.

126 Schiratzki, Body (Fn. 9), S. 3. Die Angaben beruhen auf Implementationsstudien, die das Swedish National Board of Health and Welfare in den Jahren 2005 und 2007 durchgeführt hat. Die 40-50 jährlichen neonatal durchgeführten jüdischen Beschneidungen bewegen sich dagegen innerhalb des gesetzlichen Rahmens. In einigen Fällen wurden auch muslimische Knaben durch einen zugelassenen jüdischen Mohel beschnitten. 
muslimischer Eltern in der Kommunikation mit den nationalen Gesundheitsbehörden zu sein. ${ }^{127}$ Hieraus lässt sich lernen, dass politische Kriminalisierungsdebatten ein Klima schaffen, indem auch sinnvolle Maßnahmen der Qualitätssicherung bei der Zirkumzision behindert werden. Dabei ist vorrangig an nicht-strafrechtliche Maßnahmen, wie die Entwicklung von Informationskampagnen der Gesundheitsbehörden über die Möglichkeiten sicherer, freiwilliger und informierter Beschneidungen in klinischen Settings zu denken. Dies setzt allerdings die Möglichkeit und die Bereitschaft der Kinderchirurgen und Ärzteverbände voraus, mit Eltern, die sich aus welchen Gründen auch immer für die Beschneidung ihrer Söhne entscheiden, auf Augenhöhe, in gegenseitigem Respekt und ohne Angst vor dem Staatsanwalt zu kommunizieren.

\section{E. Fazit}

Differences alter cases: Rechtswissenschaft besteht nicht nur im Common Law vornehmlich in der Kunst, Unterschiede zu machen. Das juristische Unterscheidungsvermögen beruht dabei auf der komplexen normativen Architektur, die eine Rechtsordnung evolutionär aufgebaut hat. Vor dem Hintergrund der spezifischen normativen Komplexität der deutschen Rechtsordnung lässt sich das intrikate Problem der strafrechtlichen Legitimation der Beschneidung von Knaben nicht auf eine simple objektive Kosten-Nutzen-Analyse der medizinischen Vor- und Nachteile des Eingriffs reduzieren. Die verfassungsrechtlichen Grundentscheidungen für das triadische Verhältnis von Staat, Eltern und Kindern strahlen auch auf das Strafrecht aus. Die strafrechtsdogmatischen Grenzen der stellvertretenden Einwilligung müssen das Primat der elterlichen Personensorge beachten, das aufgrund der freiheitsrechtlichen Dimension des Grundrechts auf körperliche Unversehrtheit auch für die Einwilligung in körperliche Eingriffe gilt. Für die Beschneidung von Knaben bedeutet dies, dass die primär den Sorgeberechtigten zugewiesene Abwägungsentscheidung lediglich einer Unvertretbarkeitskontrolle unterzogen wird. Die Dispositionsbefugnis der Eltern endet dort, wo sich die stellvertretende Einwilligung als gröbliche Verletzung und damit als Missbrauch der elterlichen Sorge darstellt, die zu einer Gefährdung oder Verletzung des Kindeswohls führt. Die juristische Unvertretbarkeitskontrolle berücksichtigt dabei die Schwere des Eingriffs, seine Folgen und gesundheitlichen Risiken (1), die mit dem Eingriff verbundenen (präventiv)medizinischen und sonstigen Vorteile und Chancen (2) und schließlich das Vorliegen von spezifisch kindeswohlverletzenden Eingriffsmodalitäten (Bestrafung, Demütigung, Missachtung von Vetorechten und sonstige entwürdigende Maßnahmen), die als Einwilligungssperren wirken (3). Die lege artis durchgeführte Beschneidung von Knaben stellt einen relativ leichten Eingriff ohne negative gesundheitliche Folgen und mit einem nur geringem Risiko leichter Komplikationen dar, bei der regelmäßig keine spezifisch kindeswohl- 
verletzenden Eingriffsmodalitäten vorliegen. Dem stehen nicht nur in den Fällen kurativ-medizinischer Indikation erhebliche hygienische und präventiv-medizinische Vorteile gegenüber. Religiöse Beschneidungen können daher, nicht anders als präventiv-medizinisch, hygienisch oder ästhetisch-kulturell motivierte Zirkumzisionen von Knaben durch die stellvertretende Einwilligung des Sorgeberechtigten gerechtfertigt werden, ohne dass auf die Religionsfreiheit als Rechtfertigungsgrund zurückgegriffen werden müsste. Der vorgeschlagene Prüfungsmaßstab und seine dreistufige Konkretisierung erhellen zugleich, dass die strafrechtlich unterschiedliche Behandlung der Beschneidung von Knaben und der Genitalverstümmelung bei Mädchen nicht nur legitim, sondern geboten ist. 